\title{
New emerging results in Higgs precision analysis updates 2018 after establishment of third-generation Yukawa couplings
}

\author{
Kingman Cheung, ${ }^{a, b, c, d}$ Jae Sik Lee ${ }^{a, e, f}$ and Po-Yan Tseng ${ }^{a, g}$ \\ ${ }^{a}$ Physics Division, National Center for Theoretical Sciences, Hsinchu, Taiwan \\ ${ }^{b}$ Department of Physics, National Tsing Hua University, Hsinchu 300, Taiwan \\ ${ }^{c}$ Division of Quantum Phases and Devices, School of Physics, \\ Konkuk University, Seoul 143-701, Republic of Korea \\ ${ }^{d}$ Department of Physics, National Central University, Chungli, Taiwan \\ ${ }^{e}$ Department of Physics, \\ Chonnam National University, \\ 300 Yongbong-dong, Buk-gu, Gwangju, 500-757, Republic of Korea \\ ${ }^{f}$ Institute for Universe and Elementary Particles, Chonnam National University, \\ 300 Yongbong-dong, Buk-gu, Gwangju, 500-757, Republic of Korea \\ ${ }^{g}$ Kavli IPMU (WPI), UTIAS, The University of Tokyo, \\ Kashiwa, Chiba 277-8583, Japan \\ E-mail: kingman.cheung@cern.ch, jslee@jnu.ac.kr, tpoyan1209@gmail.com
}

ABSTRACT: We perform global fits of the Higgs boson couplings to all the $7 \mathrm{TeV}, 8 \mathrm{TeV}$, and $13 \mathrm{TeV}$ data available up to the Summer 2018. New measurements at $13 \mathrm{TeV}$ extend to include the Higgs signal strengths exclusively measured in associated Higgs production with top-quark pair and the third-generation Yukawa couplings now have been established. Some important consequences emerge from the global fits. (i) The overall average signal strength of the Higgs boson stands at $2 \sigma$ above the SM value $(\mu=1.10 \pm 0.05)$. (ii) For the first time the bottom-quark Yukawa coupling shows a preference of the positive sign to the negative one. (iii) The negative top-quark Yukawa coupling is completely ruled out unless there exist additional particles running in the $H-\gamma-\gamma$ loop with contributions equal to two times the SM top-quark contribution within about $10 \%$. (iv) The branching ratio for nonstandard decays of the Higgs boson is now below $8.4 \%$ at the $95 \%$ confidence level.

KEYWORDS: Phenomenological Models

ARXIV EPRINT: 1810.02521 


\section{Contents}

1 Introduction 1

2 Formalism 2

$\begin{array}{llr}3 & \text { Results on Higgs signal strength data } & 6\end{array}$

4 Results on global fits $\quad 8$

4.1 CP conserving fits 9

4.1.1 CPC1 to CPC6 9

$\begin{array}{lll}4.1 .2 & \text { CPCN2 to CPCN4 } & 14\end{array}$

$\begin{array}{lll}\text { 4.1.3 CPCX2 to CPCX4 } & 15\end{array}$

$\begin{array}{lll}4.2 \text { CP violating fits } & 17\end{array}$

4.3 Predictions for $H \rightarrow Z \gamma \quad 20$

$\begin{array}{llr}5 & \text { Conclusions } & 20\end{array}$

A Relation between our formalism and the kappa formalism 22

B $13 \mathrm{TeV}$ data: tables 9, 10, 11, 12, 13, and 14

\section{Introduction}

Ever since the discovery of a standard model (SM) like Higgs boson in 2012 [1, 2], the main focus of the LHC experiments has been put on fully establishing its identity. Though the initial data sets till the summer 2013 indicated that it might be different from the SM Higgs boson [3], the data sets collected till the summer 2014 showed that the data is best described by the SM Higgs boson [4]. Ever since then more production channels and decay channels of the Higgs boson are established. On the production side, in addition to gluon fusion ( $\mathrm{ggF}$ ), vector-boson fusion ( $\mathrm{VBF}$ ), the associated production with a $V=W / Z$ boson $(\mathrm{VH})$, and the associated production with a top-quark pair (ttH) have been extensively investigated $[5,6]$. On the decay side, $H \rightarrow b \bar{b}[7,8]$ and $H \rightarrow \tau \tau[9,10]$ were also very recently established in single measurements. ${ }^{1}$ It is the right timing to perform the global fits to all Higgs-boson signal strengths in various scenarios of new physics, generically labeled by $\mathbf{C P C} n$ and $\mathbf{C P V} n$ in this work with $n$ standing for the number of fitting parameters.

In this work, we analyze the direct Higgs data collected at the Tevatron and the LHC adopting the formalism suggested in ref. [3] to study the impact of the established thirdgeneration Yukawa couplings on the nature of $125 \mathrm{GeV}$ Higgs. More precisely, we use 3

\footnotetext{
${ }^{1}$ We note that, in combined measurements of CMS and ATLAS, $H \rightarrow \tau \tau$ was already established in Run I [11].
} 
signal strengths measured at the Tevatron $[12,13]$ and, for the Higgs-boson data at 7 and $8 \mathrm{TeV}$, we use 20 signal strengths and the correlation matrix obtained in the combined ATLAS and CMS analysis [11]. On the other hand, for the $13 \mathrm{TeV}$ data, we use 41 signal strengths in total. Since any information on correlations between ATLAS and CMS data and those among various channels is not currently available, it is assumed that each data at $13 \mathrm{TeV}$ is Gaussian distributed and correlations among them are ignored accordingly. And, when we combine them, we simply take a $\chi^{2}$ method. For the details of the $13 \mathrm{TeV}$ data, we refer to appendix B.

Some very interesting results emerge from the new global fits, which were not realized previously.

1. The combined average signal strength of the Higgs boson now stands at a 2- $\sigma$ deviation from the SM value, namely $\mu_{\exp }=1.10 \pm 0.05$.

2. For the first time the bottom-Yukawa coupling shows statistical difference between the positive and negative signs. Thanks to the discriminating power of the Higgsgluon vertex $S^{g}$ the positive sign of the bottom-Yukawa is more preferred than the negative one.

3. Previously in 2014 the fits still allowed the negative sign of the top-Yukawa coupling at the $95 \%$ confidence level (CL). Now with more precisely measured signal strengths together with the establishment of the associated production with the top-quark pair, the negative island of the top-Yukawa is now entirely ruled out, except in the scenarios with non-zero $\Delta S^{\gamma}$. Even with $\Delta S^{\gamma} \neq 0$, it has to be adjusted within 10 $\%$ of two times the SM top-quark contribution. This tuning is going to be more and more severe as more data accumulate.

4. The nonstandard (or invisible decay) branching ratio of the Higgs boson is now reduced to less than $8.4 \%$ at the $95 \%$ CL which improves substantially from the previous value of $19 \%$. This is obtained by varying only $\Delta \Gamma_{\text {tot }}$.

The organization of the paper is as follows. In the next section, we describe briefly our formalism to make this work more self-contained. In section 3, we show the data for the Higgs signal strengths. In section 4, we show the results for all the fits. We conclude in section 5. In appendix A, we describe the correspondence between the coupling modifiers of our work with those of the LHCHXSWG $[14,15]$ and of a recent ATLAS paper [16]. In appendix B, we list all the $13 \mathrm{TeV}$ Higgs boson data that we use in our global fitting.

\section{Formalism}

In order to make the current presentation more self-contained, we include here brief description of the formalism that we use in calculating the signal strengths and chi-squares. We follow the conventions and notations of CPsuperH [17-19] for the Higgs couplings to the SM particles assuming the Higgs boson is a generally CP-mixed state without carrying any definite CP-parity. 
- Higgs couplings to fermions:

$$
\mathcal{L}_{H \bar{f} f}=-\sum_{f=u, d, l} \frac{g m_{f}}{2 M_{W}} \sum_{i=1}^{3} H \bar{f}\left(g_{H \bar{f} f}^{S}+i g_{H \bar{f} f}^{P} \gamma_{5}\right) f .
$$

For the SM couplings, $g_{H \bar{f} f}^{S}=1$ and $g_{H \bar{f} f}^{P}=0$.

- Higgs couplings to the massive vector bosons:

$$
\mathcal{L}_{H V V}=g M_{W}\left(g_{H W W} W_{\mu}^{+} W^{-\mu}+g_{H Z Z} \frac{1}{2 c_{W}^{2}} Z_{\mu} Z^{\mu}\right) H .
$$

For the SM couplings, we have $g_{H W W}=g_{H Z Z} \equiv g_{H V V}=1$, respecting the custodial symmetry.

- Higgs couplings to two photons: the amplitude for the decay process $H \rightarrow \gamma \gamma$ can be written as

$$
\mathcal{M}_{\gamma \gamma H}=-\frac{\alpha M_{H}^{2}}{4 \pi v}\left\{S^{\gamma}\left(M_{H}\right)\left(\epsilon_{1 \perp}^{*} \cdot \epsilon_{2 \perp}^{*}\right)-P^{\gamma}\left(M_{H}\right) \frac{2}{M_{H}^{2}}\left\langle\epsilon_{1}^{*} \epsilon_{2}^{*} k_{1} k_{2}\right\rangle\right\},
$$

where $k_{1,2}$ are the momenta of the two photons and $\epsilon_{1,2}$ the wave vectors of the corresponding photons, $\epsilon_{1 \perp}^{\mu}=\epsilon_{1}^{\mu}-2 k_{1}^{\mu}\left(k_{2} \cdot \epsilon_{1}\right) / M_{H}^{2}, \epsilon_{2 \perp}^{\mu}=\epsilon_{2}^{\mu}-2 k_{2}^{\mu}\left(k_{1} \cdot \epsilon_{2}\right) / M_{H}^{2}$ and $\left\langle\epsilon_{1} \epsilon_{2} k_{1} k_{2}\right\rangle \equiv \epsilon_{\mu \nu \rho \sigma} \epsilon_{1}^{\mu} \epsilon_{2}^{\nu} k_{1}^{\rho} k_{2}^{\sigma}$. The decay rate of $H \rightarrow \gamma \gamma$ is proportional to $\left|S^{\gamma}\right|^{2}+\left|P^{\gamma}\right|^{2}$. Including some additional loop contributions from new particles, the scalar and pseudoscalar form factors, retaining only the dominant loop contributions from the third-generation fermions and $W^{ \pm}$, are given by ${ }^{2}$

$$
\begin{aligned}
& S^{\gamma}\left(M_{H}\right)=2 \sum_{f=b, t, \tau} N_{C} Q_{f}^{2} g_{H \bar{f} f}^{S} F_{s f}\left(\tau_{f}\right)-g_{H W W} F_{1}\left(\tau_{W}\right)+\Delta S^{\gamma}, \\
& P^{\gamma}\left(M_{H}\right)=2 \sum_{f=b, t, \tau} N_{C} Q_{f}^{2} g_{H \bar{f} f}^{P} F_{p f}\left(\tau_{f}\right)+\Delta P^{\gamma},
\end{aligned}
$$

where $\tau_{x}=M_{H}^{2} / 4 m_{x}^{2}, N_{C}=3$ for quarks and $N_{C}=1$ for taus, respectively. The additional contributions $\Delta S^{\gamma}$ and $\Delta P^{\gamma}$ are assumed to be real in our work, as there are unlikely any new charged particles lighter than $M_{H} / 2$.

Taking $M_{H}=125.09 \mathrm{GeV}$, we find that

$$
\begin{aligned}
S^{\gamma} \simeq & -8.34 g_{H W W}+1.76 g_{H \bar{t} t}^{S}+(-0.015+0.017 i) g_{H \bar{b} b}^{S} \\
& +(-0.024+0.022 i) g_{H \bar{\tau} \tau}^{S}+(-0.007+0.005 i) g_{H \bar{c} c}^{S}+\Delta S^{\gamma} \\
P^{\gamma} \simeq & 2.78 g_{H \bar{t} t}^{P}+(-0.018+0.018 i) g_{H \bar{b} b}^{P} \\
& +(-0.025+0.022 i) g_{H \bar{\tau} \tau}^{P}+(-0.007+0.005 i) g_{H \bar{c} c}^{P}+\Delta P^{\gamma}
\end{aligned}
$$

giving $S_{\mathrm{SM}}^{\gamma}=-6.62+0.044 i$ and $P_{\mathrm{SM}}^{\gamma}=0$.

\footnotetext{
${ }^{2}$ For the loop functions of $F_{s f, p f, 1}(\tau)$, we refer to, for example, ref. [17].
} 
- Higgs couplings to two gluons: similar to $H \rightarrow \gamma \gamma$, the amplitude for the decay process $H \rightarrow g g$ can be written as

$$
\mathcal{M}_{g g H}=-\frac{\alpha_{s} M_{H}^{2} \delta^{a b}}{4 \pi v}\left\{S^{g}\left(M_{H}\right)\left(\epsilon_{1 \perp}^{*} \cdot \epsilon_{2 \perp}^{*}\right)-P^{g}\left(M_{H}\right) \frac{2}{M_{H}^{2}}\left\langle\epsilon_{1}^{*} \epsilon_{2}^{*} k_{1} k_{2}\right\rangle\right\},
$$

where $a$ and $b(a, b=1$ to 8$)$ are indices of the eight $\mathrm{SU}(3)$ generators in the adjoint representation. The decay rate of $H \rightarrow g g$ is proportional to $\left|S^{g}\right|^{2}+\left|P^{g}\right|^{2}{ }^{3}$ Again, including some additional loop contributions from new particles, the scalar and pseudoscalar form factors are given by

$$
\begin{aligned}
& S^{g}\left(M_{H}\right)=\sum_{f=b, t} g_{H \bar{f} f}^{S} F_{s f}\left(\tau_{f}\right)+\Delta S^{g}, \\
& P^{g}\left(M_{H}\right)=\sum_{f=b, t} g_{H \bar{f} f}^{P} F_{p f}\left(\tau_{f}\right)+\Delta P^{g} .
\end{aligned}
$$

The additional contributions $\Delta S^{g}$ and $\Delta P^{g}$ are assumed to be real again.

Taking $M_{H}=125.09 \mathrm{GeV}$, we find that

$$
\begin{aligned}
& S^{g} \simeq 0.688 g_{H \bar{t} t}^{S}+(-0.037+0.050 i) g_{H \bar{b} b}^{S}+\Delta S^{g}, \\
& P^{g} \simeq 1.047 g_{H \bar{t} t}^{P}+(-0.042+0.051 i) g_{H \bar{b} b}^{P}+\Delta P^{g},
\end{aligned}
$$

giving $S_{\mathrm{SM}}^{g}=0.651+0.050 i$ and $P_{\mathrm{SM}}^{g}=0$.

- Higgs couplings to $Z$ and $\gamma$ : the amplitude for the decay process $H \rightarrow$ $Z\left(k_{1}, \epsilon_{1}\right) \gamma\left(k_{2}, \epsilon_{2}\right)$ can be written as

$$
\mathcal{M}_{Z \gamma H}=-\frac{\alpha}{2 \pi v}\left\{S^{Z \gamma}\left(M_{H}\right)\left[k_{1} \cdot k_{2} \epsilon_{1}^{*} \cdot \epsilon_{2}^{*}-k_{1} \cdot \epsilon_{2}^{*} k_{2} \cdot \epsilon_{1}^{*}\right]-P^{Z \gamma}\left(M_{H}\right)\left\langle\epsilon_{1}^{*} \epsilon_{2}^{*} k_{1} k_{2}\right\rangle\right\}
$$

where $k_{1,2}$ are the momenta of the $Z$ boson and the photon (we note that $2 k_{1} \cdot k_{2}=$ $\left.M_{H}^{2}-M_{Z}^{2}\right), \epsilon_{1,2}$ are their polarization vectors. The scalar and pseudoscalar form factors can be found in ref. [3].

Finally, we define the ratios of the effective Higgs couplings to $g g, \gamma \gamma$, and $Z \gamma$ relative to the SM ones as follows:

$$
C_{g} \equiv \sqrt{\frac{\left|S^{g}\right|^{2}+\left|P^{g}\right|^{2}}{\left|S_{\mathrm{SM}}^{g}\right|^{2}}} ; \quad C_{\gamma} \equiv \sqrt{\frac{\left|S^{\gamma}\right|^{2}+\left|P^{\gamma}\right|^{2}}{\left|S_{\mathrm{SM}}^{\gamma}\right|^{2}}} ; \quad C_{Z \gamma} \equiv \sqrt{\frac{\left|S^{Z \gamma}\right|^{2}+\left|P^{Z \gamma}\right|^{2}}{\left|S_{\mathrm{SM}}^{Z}\right|^{2}}}
$$

Note that the ratios of decay rates relative to the SM are given by $\left|C_{g}\right|^{2},\left|C_{\gamma}\right|^{2}$, and $\left|C_{Z_{\gamma}}\right|^{2}$, respectively.

The theoretical signal strength may be written as the product

$$
\widehat{\mu}(\mathcal{P}, \mathcal{D}) \simeq \widehat{\mu}(\mathcal{P}) \widehat{\mu}(\mathcal{D})
$$

\footnotetext{
${ }^{3}$ Note that the production rate of $g g \rightarrow H$ at the Higgs peak is also proportional to $\left|S^{g}\right|^{2}+\left|P^{g}\right|^{2}$ in our formalism.
} 
where $\mathcal{P}=$ ggF, $\mathrm{VBF}, \mathrm{VH}, \mathrm{ttH}$ denote the production mechanisms and $\mathcal{D}=$ $\gamma \gamma, Z Z, W W, b \bar{b}, \tau \bar{\tau}$ the decay channels. More explicitly, we are taking

$$
\begin{aligned}
\widehat{\mu}(\mathrm{ggF}) & =\frac{\left|S^{g}\left(M_{H}\right)\right|^{2}+\left|P^{g}\left(M_{H}\right)\right|^{2}}{\left|S_{\mathrm{SM}}^{g}\left(M_{H}\right)\right|^{2}}, \\
\widehat{\mu}(\mathrm{VBF}) & =g_{H W W, H Z Z}^{2}, \\
\widehat{\mu}(\mathrm{VH}) & =g_{H W W, H Z Z}^{2}, \\
\widehat{\mu}(\mathrm{ttH}) & =\left(g_{H \bar{t} t}^{S}\right)^{2}+\left(g_{H \bar{t} t}^{P}\right)^{2} ;
\end{aligned}
$$

and

with

$$
\widehat{\mu}(\mathcal{D})=\frac{B(H \rightarrow \mathcal{D})}{B\left(H_{\mathrm{SM}} \rightarrow \mathcal{D}\right)}
$$

$$
B(H \rightarrow \mathcal{D})=\frac{\Gamma(H \rightarrow \mathcal{D})}{\Gamma_{\text {tot }}(H)+\Delta \Gamma_{\text {tot }}}
$$

Note that we introduce an arbitrary non-SM contribution $\Delta \Gamma_{\text {tot }}$ to the total decay width. Incidentally, $\Gamma_{\text {tot }}(H)$ becomes the SM total decay width when $g_{H \bar{f} f}^{S}=1, g_{H \bar{f} f}^{P}=0$, $g_{H W W, H Z Z}=1, \Delta S^{\gamma, g, Z \gamma}=\Delta P^{\gamma, g, Z \gamma}=0$.

The experimentally observed signal strengths should be compared to the theoretical ones summed over all production mechanisms:

$$
\mu(\mathcal{Q}, \mathcal{D})=\sum_{\mathcal{P}=\mathrm{ggF}, \mathrm{VBF}, \mathrm{VH}, \mathrm{ttH}} C_{\mathcal{Q P}} \widehat{\mu}(\mathcal{P}, \mathcal{D})
$$

where $\mathcal{Q}$ denote the experimentally defined channel involved with the decay $\mathcal{D}$ and the decomposition coefficients $C_{\mathcal{Q P}}$ may depend on the relative Higgs production cross sections for a given Higgs-boson mass, experimental cuts, etc.

The $\chi^{2}$ associated with an uncorrelated observable is

$$
\chi^{2}(\mathcal{Q}, \mathcal{D})=\frac{\left[\mu(\mathcal{Q}, \mathcal{D})-\mu^{\mathrm{EXP}}(\mathcal{Q}, \mathcal{D})\right]^{2}}{\left[\sigma^{\mathrm{EXP}}(\mathcal{Q}, \mathcal{D})\right]^{2}},
$$

where $\sigma^{\operatorname{EXP}}(\mathcal{Q}, \mathcal{D})$ denotes the experimental error. For $n$ correlated observables, we use

$$
\chi_{n}^{2}=\sum_{i, j=1}^{n}\left(\mu_{i}-\mu_{i}^{\mathrm{EXP}}\right)\left(V^{-1}\right)_{i j}\left(\mu_{j}-\mu_{j}^{\mathrm{EXP}}\right),
$$

where $V$ is a $n \times n$ covariance matrix whose $(i, j)$ component is given by

$$
V_{i j}=\rho_{i j} \sigma_{i}^{\mathrm{EXP}} \sigma_{j}^{\mathrm{EXP}}
$$

with $\rho$ denoting the relevant $n \times n$ correlation matrix. Note $\rho_{i j}=\rho_{j i}, \rho_{i i}=1$, and if $\rho_{i j}=\delta_{i j}, \chi_{n}^{2}$ reduces to

$$
\chi_{n}^{2}=\sum_{i=1}^{n} \frac{\left(\mu_{i}-\mu_{i}^{\mathrm{EXP}}\right)^{2}}{\left(\sigma_{i}^{\mathrm{EXP}}\right)^{2}},
$$

i.e., the sum of $\chi^{2}$ of each uncorrelated observable. 


\begin{tabular}{|c|c|c|c|c|c|c|c|}
\hline \multirow[t]{2}{*}{ Channel } & \multirow{2}{*}{$\begin{array}{l}\text { Signal strength } \mu \\
\text { c.v } \pm \text { error }\end{array}$} & \multirow[t]{2}{*}{$M_{H}(\mathrm{GeV})$} & \multicolumn{4}{|c|}{ Production mode } & \multirow[t]{2}{*}{$\chi_{\mathrm{SM}}^{2}($ each $)$} \\
\hline & & & $\operatorname{ggF}$ & VBF & $\mathrm{VH}$ & $\mathrm{ttH}$ & \\
\hline \multicolumn{8}{|c|}{ Tevatron (Nov. 2012) } \\
\hline Combined $H \rightarrow \gamma \gamma[12]$ & $6.14_{-3.19}^{+3.25}$ & 125 & $78 \%$ & $5 \%$ & $17 \%$ & - & 2.60 \\
\hline Combined $H \rightarrow W W^{(*)}[12]$ & $0.85_{-0.81}^{+0.88}$ & 125 & $78 \%$ & $5 \%$ & $17 \%$ & - & 0.03 \\
\hline $\mathrm{VH} \operatorname{tag} H \rightarrow b b[13]$ & $1.59_{-0.72}^{+0.69}$ & 125 & - & - & $100 \%$ & - & 0.67 \\
\hline & & & & & & & btot): 3.30 \\
\hline
\end{tabular}

Table 1. (Tevatron: $1.96 \mathrm{TeV}$ ) The signal strengths data from Tevatron $\left(10.0 \mathrm{fb}^{-1}\right.$ at $\left.1.96 \mathrm{TeV}\right)$.

\begin{tabular}{|c|ccccc|}
\hline & \multicolumn{5}{|c|}{ Decay mode } \\
\hline Production mode & $H \rightarrow \gamma \gamma$ & $H \rightarrow Z Z^{(*)}$ & $H \rightarrow W W^{(*)}$ & $H \rightarrow b b$ & $H \rightarrow \tau^{+} \tau^{-}$ \\
\hline ggF & $1.10_{-0.22}^{+0.23}$ & $1.13_{-0.31}^{+0.34}$ & $0.84_{-0.17}^{+0.17}$ & - & $1.0_{-0.6}^{+0.6}$ \\
VBF & $1.3_{-0.5}^{+0.5}$ & $0.1_{-0.6}^{+1.1}$ & $1.2_{-0.4}^{+0.4}$ & - & $1.3_{-0.4}^{+0.4}$ \\
WH & $0.5_{-1.2}^{+1.3}$ & - & $1.6_{-1.0}^{+1.2}$ & $1.0_{-0.5}^{+0.5}$ & $-1.4_{-1.4}^{+1.4}$ \\
ZH & $0.5_{-2.5}^{+3.0}$ & - & $5.9_{-2.2}^{+2.6}$ & $0.4_{-0.4}^{+0.4}$ & $2.2_{-1.8}^{+2.2}$ \\
$\mathrm{ttH}$ & $2.2_{-1.3}^{+1.6}$ & - & $5.0_{-1.7}^{+1.8}$ & $1.1_{-1.0}^{+1.0}$ & $-1.9_{-3.3}^{+3.7}$ \\
\hline & & & & & $\chi_{\text {SM }}^{2}($ subtot $): 19.93$ \\
\hline
\end{tabular}

Table 2. ( $L H C: 7+8 T e V)$ Combined ATLAS and CMS data on signal strengths from table 8 of ref. [11].

\begin{tabular}{|c|c|c|c|c|c|c|c|}
\hline & \multicolumn{5}{|c|}{ Decay mode } & \multirow[b]{2}{*}{$\mu_{\text {combined }}^{\text {prod }}$} & \multirow[b]{2}{*}{$\chi_{\mathrm{SM}}^{2}\left(\chi_{\min }^{2}\right)$} \\
\hline Production mode & $H \rightarrow \gamma \gamma$ & $H \rightarrow Z Z^{(*)}$ & $H \rightarrow W W^{(*)}$ & $H \rightarrow b b$ & $H \rightarrow \tau^{+} \tau^{-}$ & & \\
\hline $\operatorname{ggF}$ & $1.02_{-0.11}^{+0.12}$ & $1.09_{-0.11}^{+0.11}$ & $1.29_{-0.16}^{+0.16}$ & $2.51_{-2.01}^{+2.43}$ & $1.06_{-0.37}^{+0.40}$ & $1.11_{-0.07}^{+0.07}$ & $5.42(3.15)$ \\
\hline VBF & $1.23_{-0.31}^{+0.32}$ & $1.51_{-0.59}^{+0.59}$ & $0.54_{-0.31}^{+0.32}$ & - & $1.15_{-0.34}^{+0.36}$ & $1.02_{-0.18}^{+0.18}$ & $7.53(7.51)$ \\
\hline VH/WH & $1.42_{-0.51}^{+0.51}$ & $0.71_{-0.65}^{+0.65}$ & $3.27_{-1.70}^{+1.88}$ & $1.07_{-0.22}^{+0.23}$ & $3.39_{-1.54}^{+1.68}$ & $1.15_{-0.19}^{+0.20}$ & $7.05(6.44)$ \\
\hline $\mathrm{ZH}$ & - & - & $1.00_{-1.00}^{+1.57}$ & $1.20_{-0.31}^{+0.33}$ & $1.23_{-1.35}^{+1.62}$ & $1.19_{-0.30}^{+0.32}$ & $0.45(0.02)$ \\
\hline $\mathrm{ttH}$ & $1.36_{-0.37}^{+0.38}$ & $0.00_{-0.00}^{+0.53}$ & - & $0.91_{-0.43}^{+0.45}$ & - & $0.93_{-0.24}^{+0.24}$ & $5.96(5.86)$ \\
\hline ttH (excl.) & $1.39_{-0.42}^{+0.48}$ & - & $1.59_{-0.43}^{+0.44}$ & $0.77_{-0.35}^{+0.36}$ & $0.87_{-0.73}^{+0.73}$ & $1.16_{-0.22}^{+0.22}$ & $4.17(3.62)$ \\
\hline$\mu_{\text {combined }}^{\mathrm{dec}}$ & $1.10_{-0.10}^{+0.10}$ & $1.05_{-0.11}^{+0.11}$ & $1.20_{-0.13}^{+0.14}$ & $1.05_{-0.19}^{+0.19}$ & $1.15_{-0.23}^{+0.24}$ & $1.10_{-0.06}^{+0.06}$ & \\
\hline$\chi_{\mathrm{SM}}^{2}\left(\chi_{\min }^{2}\right)$ & $6.83(5.72)$ & $9.13(8.88)$ & $9.48(7.32)$ & $1.56(1.51)$ & $3.58(3.20)$ & & $30.58(27.56)$ \\
\hline
\end{tabular}

Table 3. (LHC: $13 \mathrm{TeV}$ ) Combined ATLAS and CMS (13 TeV) data on signal strengths. The $\mu_{\text {combined }}^{\text {dec }}\left(\mu_{\text {combined }}^{\text {prod }}\right)$ represents the combined signal strength for a specific decay (production) channel by summing all the production (decay) modes, and $\chi_{\min }^{2}$ are the corresponding minimal chi-square values. In the $\mathrm{VH} / \mathrm{WH}$ row, the production mode for $H \rightarrow \gamma \gamma$ and $H \rightarrow Z Z^{(*)}$ is $\mathrm{VH}$ while it is $\mathrm{WH}$ for $H \rightarrow W W^{(*)}$ and $H \rightarrow \tau^{+} \tau^{-}$; for the remaining decay mode $H \rightarrow b \bar{b}$, we combine the two signal strengths from WH and VH, see table 12.

\section{Results on Higgs signal strength data}

In our work, we use the direct Higgs data collected at the Tevatron and the LHC. We use 3 signal strengths measured at the Tevatron, see table 1 . The Higgs-boson data at 7 and 8 $(7+8) \mathrm{TeV}$ used in this analysis are the signal strengths obtained from a combined ATLAS and CMS analysis [11], see table 2. We also take into account the correlation matrix given in figure 27 of ref. [11]. On the other hand, the $13 \mathrm{TeV}$ data are still given separately by 
ATLAS and CMS and in different production and decay channels. ${ }^{4}$ In total, the number of signal strengths considered is $3(1.96 \mathrm{TeV})+20(7+8 \mathrm{TeV})+41(13 \mathrm{TeV})=64$.

Precaution is noted before we show the combined results of ATLAS and CMS. In each of the data, there are statistical, systematic, and theoretical uncertainties. Especially, the latter one, e.g., uncertainties coming from factorization scale, renormalization scale, higher order corrections, is correlated between ATLAS and CMS. Since no such information is available at the time of writing, we only combine them with a simple $\chi^{2}$ method and assuming each data is Gaussian distributed. Our finding of 2 sigma excess in the overall signal strength is to be taken cautiously.

At $7+8 \mathrm{TeV}$, we use the combined average signal strengths given in ref. [11] in which the experimental correlations are considered. At $1.96 \mathrm{TeV}$ and $13 \mathrm{TeV}$, we combine signal strengths of various channels using a simple $\chi^{2}$ method and assuming each is Gaussian distributed. The combined signal stregnth at $1.96 \mathrm{TeV}$ is $1.44 \pm 0.55$ and, at $13 \mathrm{TeV}$, the combined ATLAS and CMS signal strengths for each production and decay channel are presented in table 3. Before we go to the global fits, we would like to point out a few peculiar features in the data sets, and the average signal strengths.

1. The combined overall signal strength at $7+8 \mathrm{TeV}$ is $\mu_{7+8 \mathrm{TeV}}=1.09_{-0.10}^{+0.11}$ [11], which is larger than the SM value by slightly less than $1 \sigma$.

2. At $13 \mathrm{TeV}$, from table 3 , it is clear that all decay channels show slight excess over the SM value of 1.0, especially the $H \rightarrow \gamma \gamma$ and $H \rightarrow W W^{*}$ channels.

3. Again from table 3, almost all production modes, except for $t \mathrm{tH}$, show excess above the SM, especially the gluon fusion ( $\mathrm{ggF})$.

4. The $13 \mathrm{TeV}$ data shows similar deviations in both ATLAS and CMS results: $\mu_{13 \mathrm{TeV}}^{\mathrm{ATLAS}}=1.09 \pm 0.08$ and $\mu_{13 \mathrm{TeV}}^{\mathrm{CMS}}=1.1_{-0.08}^{+0.09}$. By combining these two results we obtain

$$
\mu_{13 \mathrm{TeV}}=1.10 \pm 0.06
$$

which is about $1.67 \sigma$ above the SM.

5. Finally, we combine all the signal strengths for the Tevatron at $1.96 \mathrm{TeV}$, and for $7+8$ and $13 \mathrm{TeV}$ ATLAS and CMS, and thus obtain

$$
\mu_{\mathrm{All}}=1.10 \pm 0.05
$$

which indicates a $2 \sigma$ deviation from the SM value.

We summarize the results in table 4 .

Here and in the following section, we will present two statistical measures: (i) goodness of fit quantifying the agreement within observables in a given fit, and (ii) $p$-value of a given

\footnotetext{
${ }^{4}$ For the details of the $13 \mathrm{TeV}$ data sets used in this work, see appendix B.
} 


\begin{tabular}{|c|c|c|c|}
\hline Energy & ATLAS & CMS & Combined \\
\hline $1.96 \mathrm{TeV}$ [table 1] & & & $1.44 \pm 0.55$ \\
\hline $7+8 \mathrm{TeV}[11]$ & $1.20_{-0.14}^{+0.15}$ & $0.97_{-0.13}^{+0.14}$ & $1.09_{-0.10}^{+0.11}$ \\
\hline $13 \mathrm{TeV}[$ table 3] & $1.09 \pm 0.08$ & $1.11_{-0.08}^{+0.09}$ & $1.10 \pm 0.06$ \\
\hline & & & $1.10 \pm 0.05$ \\
\hline
\end{tabular}

Table 4. Combined average signal strengths for the Tevatron at $1.96 \mathrm{TeV}$, and for ATLAS and CMS at $7+8 \mathrm{TeV}$ and $13 \mathrm{TeV}$.

fit hypothesis against the SM null hypothesis. Goodness of fit is expressed in terms of an integral, which is given by

$$
\text { Goodness of fit }=\int_{\chi^{2}}^{\infty} f[x, n] d x
$$

where the probability density function is given by

$$
f[x, n]=\frac{x^{n / 2-1} e^{-x / 2}}{2^{n / 2} \Gamma(n / 2)},
$$

$n$ is the degree of freedom, and $\Gamma(n / 2)$ is the gamma function. The rule of thumb is that when the value of $\chi^{2}$ per degree of freedom is less than around 1 , it is a good fit.

On the other hand, the $p$-value of the given fit hypothesis (test hypothesis) with $m$ fitting parameters against the SM null hypothesis is given by

$$
p \text {-value }=\int_{\Delta \chi^{2}}^{\infty} f[x, m] d x
$$

where $\Delta \chi^{2}$ in the lower limit of the integral is equal to chi-square difference between the best-fit point of the fit hpothesis and the SM one: $\Delta \chi^{2}=\chi_{\mathrm{SM}}^{2}-\chi_{\min }^{2}$. This $p$ value represents the probability that the test hypothesis is a fluctuation of the SM null hypothesis. A large $p$-value means that the test hypothesis is very similar to the SM null hypothesis. For example, in table 5 the CPC1 case (with 1 fitted parameter) has a $\Delta \chi^{2}=53.81-51.44=2.37$ corresponding to a $p$-value of 0.124 . For CPC2 case (with 2 fitted parameters) has $\Delta \chi^{2}=53.81-51.87=1.94$ corresponding to a $p$-value of 0.379 . From these two fits we can easily see that the SM null hypothesis is more similar to the CPC2 best fit-point. According to the $p$-values in table 5, the SM is more consistent with the fits with more parameters.

\section{Results on global fits}

We perform global fits in which one or more parameters are varied. They are categorized into CP-conserving (CPC) and CP-violating (CPV) fits, because the current data still allows the observed Higgs boson to be a mixture of CP-even and CP-odd states. Assuming generation independence for the normalized Yukawa couplings of $g_{H \bar{f} f}^{S, P}$, we use the following notation for the parameters in the fits:

$$
\begin{array}{llll}
C_{u}^{S}=g_{H \bar{u} u}^{S}, & C_{d}^{S}=g_{H \bar{d} d}^{S}, & C_{\ell}^{S}=g_{H \bar{l} l}^{S} ; & C_{w}=g_{H W W}, \quad C_{z} \quad=g_{H Z Z} ; \\
C_{u}^{P}=g_{H \bar{u} u}^{P}, & C_{d}^{P}=g_{H \bar{d} d}^{P}, & C_{\ell}^{P}=g_{H \bar{l} l}^{P} . &
\end{array}
$$


In most of the fits, we keep the custodial symmetry between the $W$ and $Z$ bosons by taking $C_{v} \equiv C_{w}=C_{z}$. However, in the last CP-conserving scenario (CPCX4), we adopt $C_{w} \neq C_{z}$, which is motivated by the data.

\subsection{CP conserving fits}

In CP-conserving fits, we are varying $C_{u}^{S}, C_{d}^{S}, C_{\ell}^{S}, C_{v ; w, z}, \Delta S^{g}, \Delta S^{\gamma}$, and $\Delta \Gamma_{\text {tot }}$ while taking $C_{u}^{P}=C_{d}^{P}=C_{\ell}^{P}=\Delta P^{\gamma}=\Delta P^{g}=0$. All the CP-conserving fits considered in this work are listed here:

- CPC1: vary $\Delta \Gamma_{\text {tot }}$ while keeping $C_{u}^{S}=C_{d}^{S}=C_{\ell}^{S}=C_{v}=1$ and $\Delta S^{\gamma}=\Delta S^{g}=0$.

- CPC2: vary $\Delta S^{\gamma}$ and $\Delta S^{g}$ while keeping $C_{u}^{S}=C_{d}^{S}=C_{\ell}^{S}=C_{v}=1$ and $\Delta \Gamma_{\text {tot }}=0$.

- CPC3: vary $\Delta S^{\gamma}, \Delta S^{g}$ and $\Delta \Gamma_{\text {tot }}$ while keeping $C_{u}^{S}=C_{d}^{S}=C_{\ell}^{S}=C_{v}=1$.

- CPC4: vary $C_{u}^{S}, C_{d}^{S}, C_{\ell}^{S}, C_{v}$ while keeping $\Delta S^{\gamma}=\Delta S^{g}=\Delta \Gamma_{\text {tot }}=0$.

- CPC6: vary $C_{u}^{S}, C_{d}^{S}, C_{\ell}^{S}, C_{v}, \Delta S^{\gamma}, \Delta S^{g}$ while keeping $\Delta \Gamma_{\text {tot }}=0$.

- CPCN2: vary $C_{u}^{S}, C_{v}$ while keeping $C_{d}^{S}=C_{\ell}^{S}=1$, and $\Delta S^{\gamma}=\Delta S^{g}=\Delta \Gamma_{\text {tot }}=0$.

- CPCN3: vary $C_{u}^{S}, C_{v}, \Delta S^{\gamma}$ while keeping $C_{d}^{S}=C_{\ell}^{S}=1$ and $\Delta S^{g}=\Delta \Gamma_{\text {tot }}=0$.

- CPCN4: vary $C_{u}^{S}, C_{v}, \Delta S^{\gamma}, \Delta S^{g}$ while keeping $C_{d}^{S}=C_{\ell}^{S}=1$ and $\Delta \Gamma_{\text {tot }}=0$.

- CPCX2: vary $C_{v}, \Delta \Gamma_{\text {tot }}$ while keeping $C_{u}^{S}=C_{d}^{S}=C_{\ell}^{S}=1$, and $\Delta S^{\gamma}=\Delta S^{g}=0$.

- CPCX3: vary $C_{u}^{S}, C_{v}, \Delta S^{g}$ while keeping $C_{d}^{S}=C_{\ell}^{S}=1$ and $\Delta S^{\gamma}=\Delta \Gamma_{\text {tot }}=0$.

- CPCX4: vary $C_{u}^{S}, C_{w}, C_{z}, \Delta S^{g}$ while keeping $C_{d}^{S}=C_{\ell}^{S}=1$ and $\Delta S^{\gamma}=\Delta \Gamma_{\text {tot }}=0$.

Note that CPC1 to CPC6 were those originally in our first Higgcision paper [3] while CPCN2 to CPCN4 were those studied in our 2014 update paper [4]. The CPCX2 to CPCX4 are new in this work. The reason why we study more scenarios here is because we want to fully understand the effects of having $\Delta S^{g}$ alone, in order to discriminate the contribution from the bottom-Yukawa coupling to Higgs production. In doing so we find that the effect of the bottom-Yukawa coupling becomes sizable in the Higgs-gluon-gluon vertex: numerically flipping the sign of bottom-Yukawa coupling can cause more than $10 \%$ change in $\left|S^{g}\right|$ while it is less than $0.5 \%$ in $\left|S^{\gamma}\right|$.

\subsubsection{CPC1 to CPC6}

The fitting results for CPC1 to CPC6 are shown in table 5. The corresponding figures for confidence regions are depicted in figure 1 to figure 5 . In the following, we are going through each fit one by one.

In CPC1, the best-fit value for $\Delta \Gamma_{\text {tot }}$ is

$$
\Delta \Gamma_{\text {tot }}=-0.285_{-0.17}^{+0.18} \mathrm{MeV}
$$




\begin{tabular}{|c|c|c|c|c|c|}
\hline Cases & CPC1 & CPC2 & CPC3 & CPC4 & CPC6 \\
\hline Parameters & Vary $\Delta \Gamma_{\text {tot }}$ & $\begin{array}{l}\text { Vary } \Delta S^{\gamma} \\
\qquad \Delta S^{g}\end{array}$ & $\begin{array}{c}\text { Vary } \Delta S^{\gamma} \\
\Delta S^{g}, \Delta \Gamma_{\text {tot }}\end{array}$ & $\begin{array}{c}\text { Vary } C_{u}^{S}, C_{d}^{S} \\
\quad C_{\ell}^{S}, C_{v}\end{array}$ & $\begin{array}{c}\text { Vary } C_{u}^{S}, C_{d}^{S}, C_{\ell}^{S}, C_{v} \\
\Delta S^{\gamma}, \Delta S^{g}\end{array}$ \\
\hline \multicolumn{6}{|c|}{ After ICHEP 2018} \\
\hline$C_{u}^{S}$ & 1 & 1 & 1 & $1.001_{-0.055}^{+0.056}$ & $1.033_{-0.082}^{+0.079}$ \\
\hline$C_{d}^{S}$ & 1 & 1 & 1 & $0.962_{-0.101}^{+0.101}$ & $0.945_{-0.105}^{+0.109}$ \\
\hline$C_{\ell}^{S}$ & 1 & 1 & 1 & $1.024_{-0.093}^{+0.093}$ & $1.018_{-0.094}^{+0.095}$ \\
\hline$C_{v}$ & 1 & 1 & 1 & $1.019_{-0.045}^{+0.044}$ & $1.012_{-0.048}^{+0.047}$ \\
\hline$\Delta S^{\gamma}$ & 0 & $-0.226_{-0.32}^{+0.32}$ & $-0.150_{-0.33}^{+0.32}$ & 0 & $-0.128_{-0.369}^{+0.368}$ \\
\hline$\Delta S^{g}$ & 0 & $0.016_{-0.025}^{+0.025}$ & $-0.003_{-0.031}^{+0.034}$ & 0 & $-0.032_{-0.057}^{+0.061}$ \\
\hline$\Delta \Gamma_{\text {tot }}(\mathrm{MeV})$ & $-0.285_{-0.17}^{+0.18}$ & 0 & $-0.247_{-0.27}^{+0.31}$ & 0 & 0 \\
\hline$\chi^{2} / d o f$ & $51.44 / 63$ & $51.87 / 62$ & $51.23 / 61$ & $50.79 / 60$ & $50.46 / 58$ \\
\hline goodness of fit & 0.851 & 0.817 & 0.809 & 0.796 & 0.749 \\
\hline$p$-value & 0.124 & 0.379 & 0.461 & 0.554 & 0.764 \\
\hline
\end{tabular}

Table 5. CPC: the best-fitted values in various CP conserving fits and the corresponding chisquare per degree of freedom and goodness of fit. The $p$-value for each fit hypothesis against the $\mathrm{SM}$ null hypothesis is also shown. For the SM, we obtain $\chi^{2}=53.81, \chi^{2} / \operatorname{dof}=53.81 / 64$, and so the goodness of fit $=0.814$.

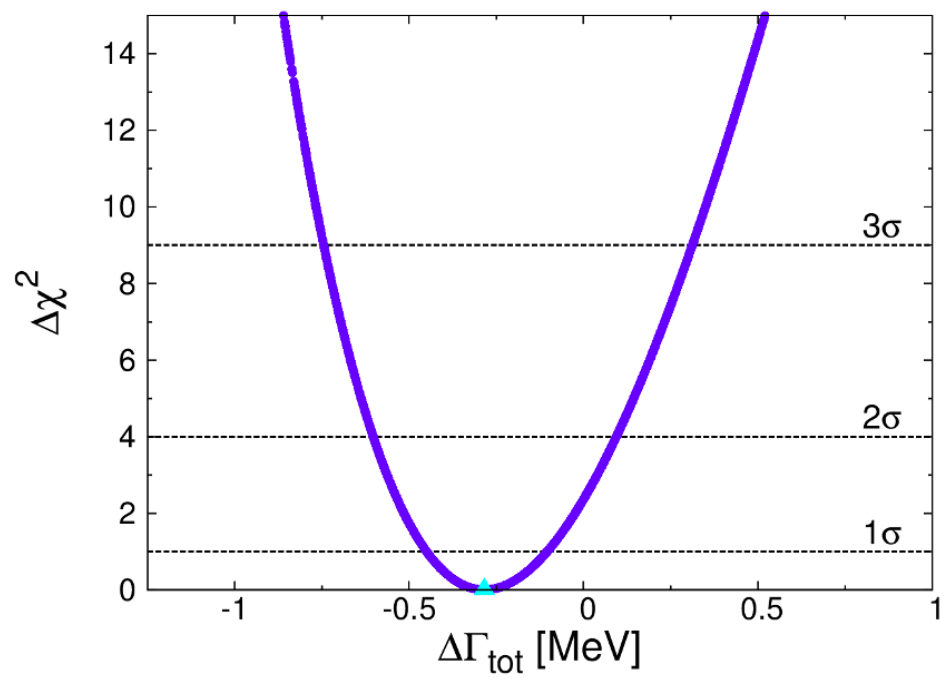

Figure 1. CPC1: $\Delta \chi^{2}$ from the minimum versus $\Delta \Gamma_{\text {tot }}$ with only $\Delta \Gamma_{\text {tot }}$ varying in the fit. The best-fit point is denoted by the triangle.

which is $1.6 \sigma$ below zero. The $p$-value of this fit is 0.851 , which is indeed better than the SM ( $p$-value $=0.814)$. This finding is consistent with the average signal strength $\mu_{\text {All }}=1.10 \pm 0.05$. Nevertheless, we do not recall any new physics models that reduce the total decay width. From the fit we can determine the upper limit for $\Delta \Gamma_{\text {tot }}$. The $95 \%$ CL allowed range for $\Delta \Gamma_{\text {tot }}=-0.285_{-0.32}^{+0.38}$, as shown in figure 1. Assuming the fit is consistent with the SM, the $95 \%$ CL upper limit for $\Delta \Gamma_{\text {tot }}=0.38 \mathrm{MeV}$ (we simply take the central value equal to zero and use the upper error as the upper limit), which translates to 

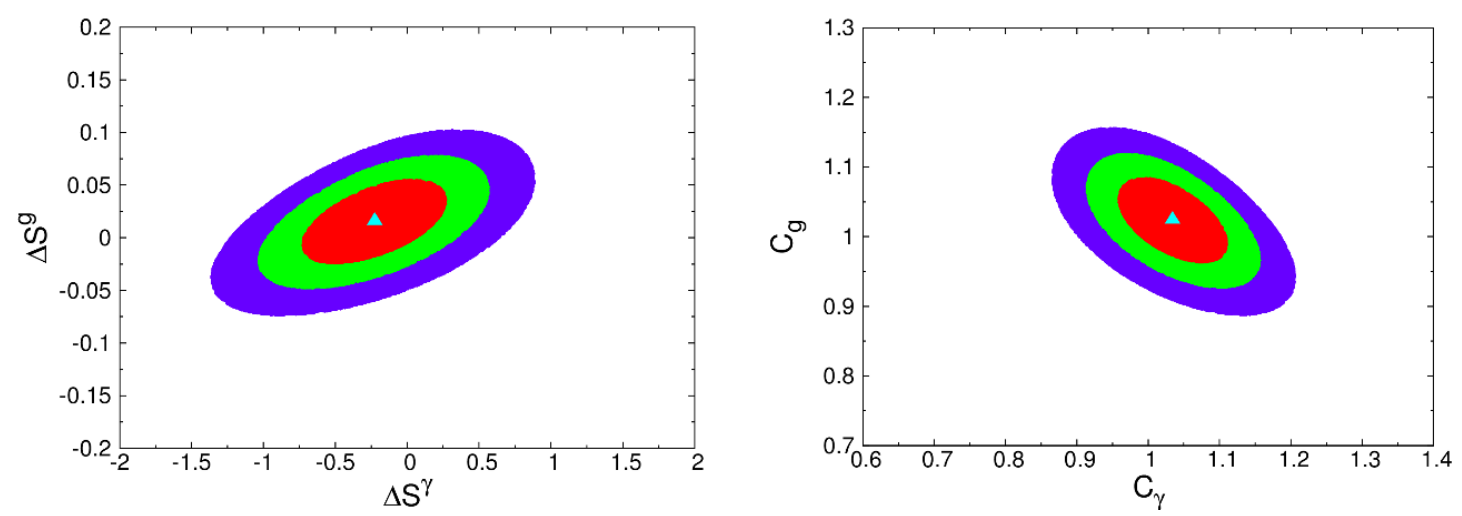

Figure 2. CPC2: the confidence-level regions of the fit by varying $\Delta S^{\gamma}$ and $\Delta S^{g}$ only in (a) $\left(\Delta S^{\gamma}, \Delta S^{g}\right)$ plane and (b) in the corresponding $\left(C_{\gamma}, C_{g}\right)$ plane. The contour regions shown are for $\Delta \chi^{2} \leq 2.3$ (red), 5.99 (green), and 11.83 (blue) above the minimum, which correspond to confidence levels of $68.3 \%, 95 \%$, and $99.7 \%$, respectively. The best-fit point is denoted by the triangle.

a branching ratio

$$
B(H \rightarrow \text { nonstandard })<8.4 \%,
$$

which improves significantly from the previous value of $19 \%$ [4].

In CPC2, we vary $\Delta S^{g}$ and $\Delta S^{\gamma}$ - the vertex factors for $H g g$ and $H \gamma \gamma$, respectively. This scenario accounts for additional charged particles running in the loop of $H \gamma \gamma$ vertex and additional colored particles running in the loop of $H g g$ vertex. The best-fit point $\left(\Delta S^{\gamma}, \Delta S^{g}\right)=(-0.226,0.016)$ shows an increase of $3.4 \%$ and $2.4 \%$ in $\left|S^{\gamma}\right|$ and $\left|S^{g}\right|$, respectively. We note that the error of $\Delta S^{g}$ is now \pm 0.025 , which is numerically smaller than the SM bottom-quark contribution of -0.037 to the real part of $S^{g}$, see eq. (2.8), alerting that we have reached the sensitivity to probe the sign of the bottom-quark Yukawa coupling in gluon fusion. The $p$-value of the best-fit point is about as the SM one. In figure 2, we show the confidence-level regions of the fit for $\Delta \chi^{2} \leq 2.3$ (red), 5.99 (green), and 11.83 (blue) above the minimum, which correspond to confidence levels of $68.3 \%, 95 \%$, and $99.7 \%$, respectively. The corresponding regions for $\left(C^{\gamma}, C^{g}\right)$ are also shown in the right panel.

In CPC3, $\Delta \Gamma_{\text {tot }}, \Delta S^{g}$, and $\Delta S^{\gamma}$ are the varying parameters. The best-fit point shows that the data prefer modification of $\Delta \Gamma_{\text {tot }}$ to accommodate the data rather than the other two parameters. It implies that the excesses are seen in most channels, not just the diphoton channel. Nevertheless, the $p$-value of this fit is very similar to CPC2 and the SM. On the other hand, the better $p$-value of CPC1 indicates that the data prefer enhancement in all channels, instead of a particular one. The confidence-level regions of the fit are shown in figure 3.

The CPC4 fit allows $C_{v}, C_{u}^{S}, C_{d}^{S}, C_{\ell}^{S}$ to vary, and it shows two most dramatic changes from previous results [3, 4]. (i) The "island" on the negative of $C_{u}^{S}$ in the $\left(C_{u}^{S}, C_{v}\right)$ plane completely disappears, shown in the left panels of figure 4. (ii) The middle panels show that $C_{d}^{S}$ now prefers the positive sign to the negative one. It is more clear from the middle-lower panel that the point $C_{d}^{S}=-1$ has $\Delta \chi^{2}>2$ above the minimum at $C_{d}^{S}=$ +1 . This is the first time that the data prefer positive bottom-Yukawa coupling to the negative one. The key observation here is that when we change the sign of bottom-Yukawa 

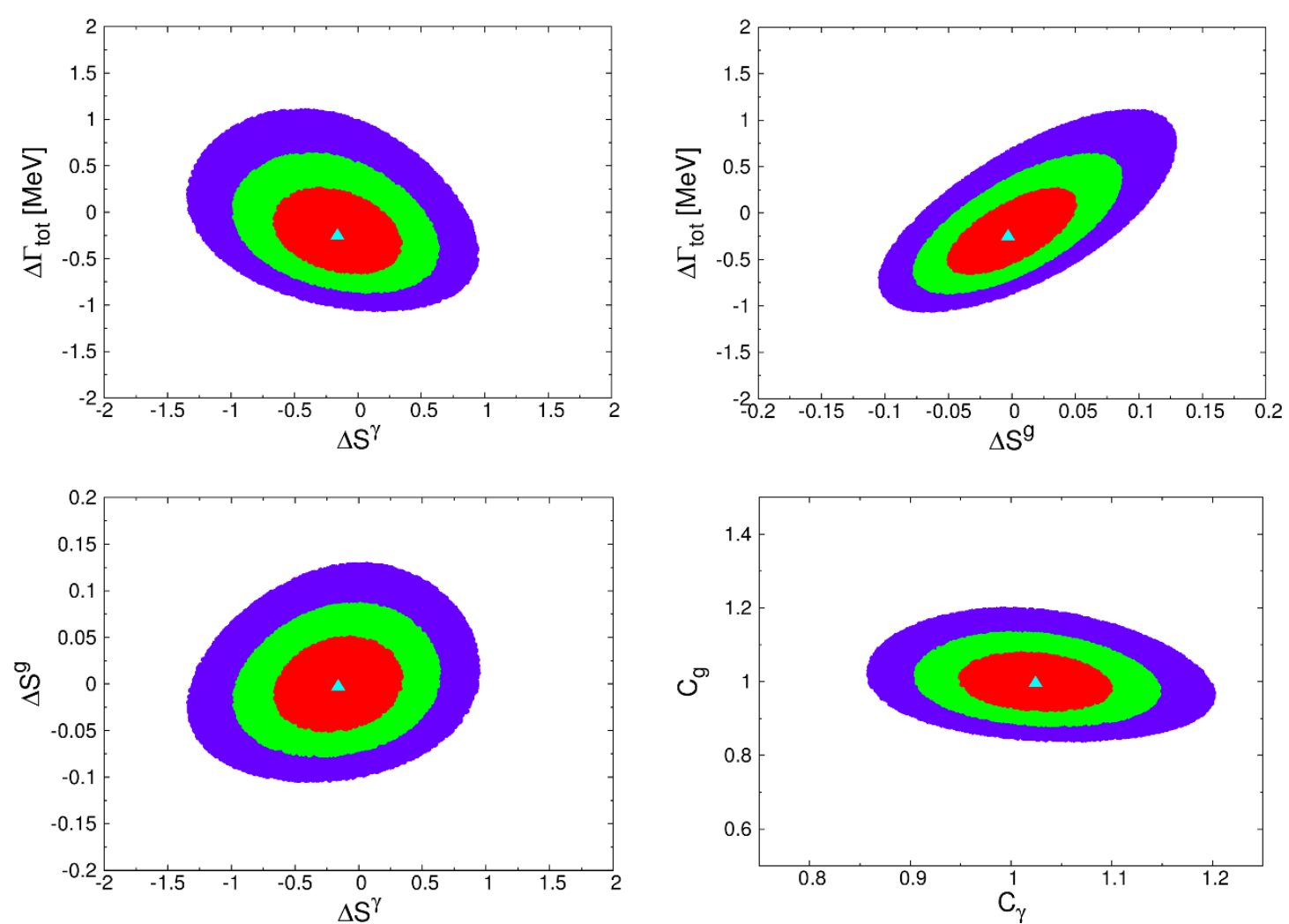

Figure 3. CPC3: the confidence-level regions of the fit by varying $\Delta S^{\gamma}, \Delta S^{g}$, and $\Delta \Gamma_{\text {tot }}$. The color code is the same as in figure 2 .
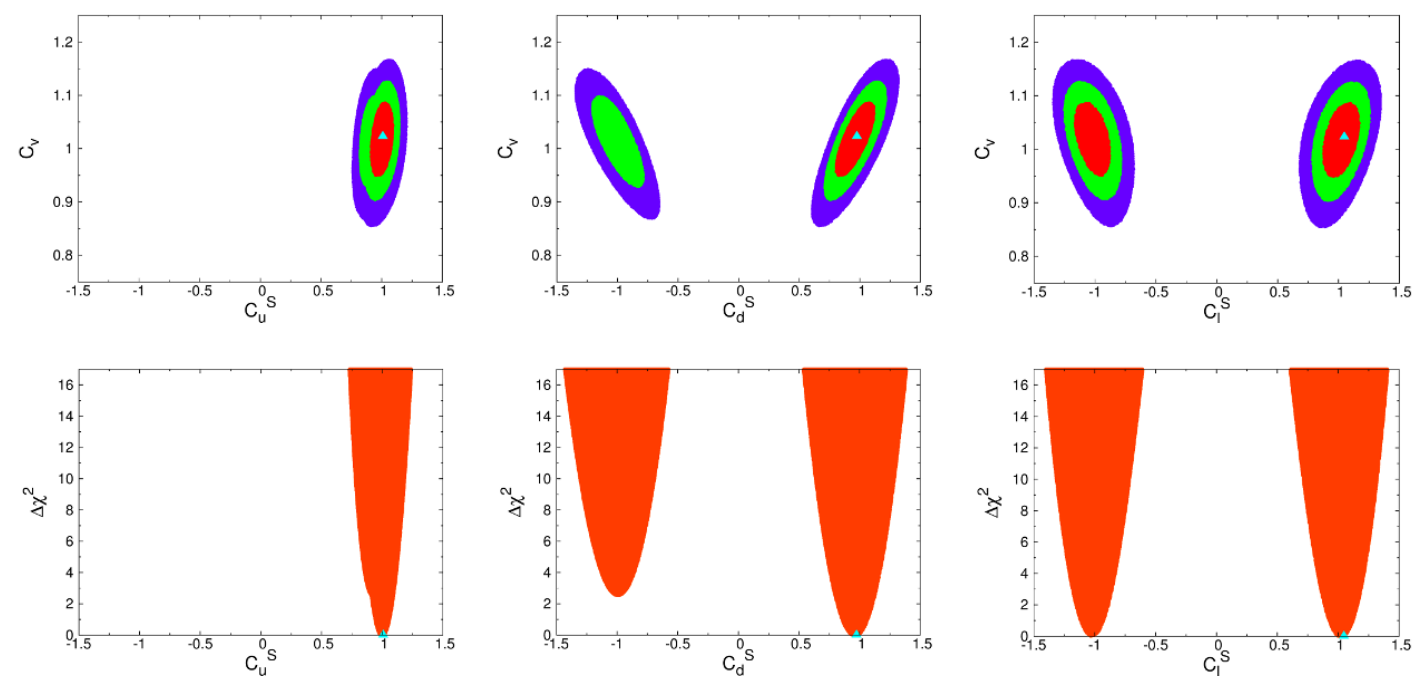

Figure 4. CPC4: (Upper) The confidence-level regions of the fit by varying $C_{v}, C_{u}^{S}, C_{d}^{S}$, and $C_{\ell}^{S}$. The color code is the same as figure 2. (Lower) $\Delta \chi^{2}$ from the minimum versus Yukawa couplings. 

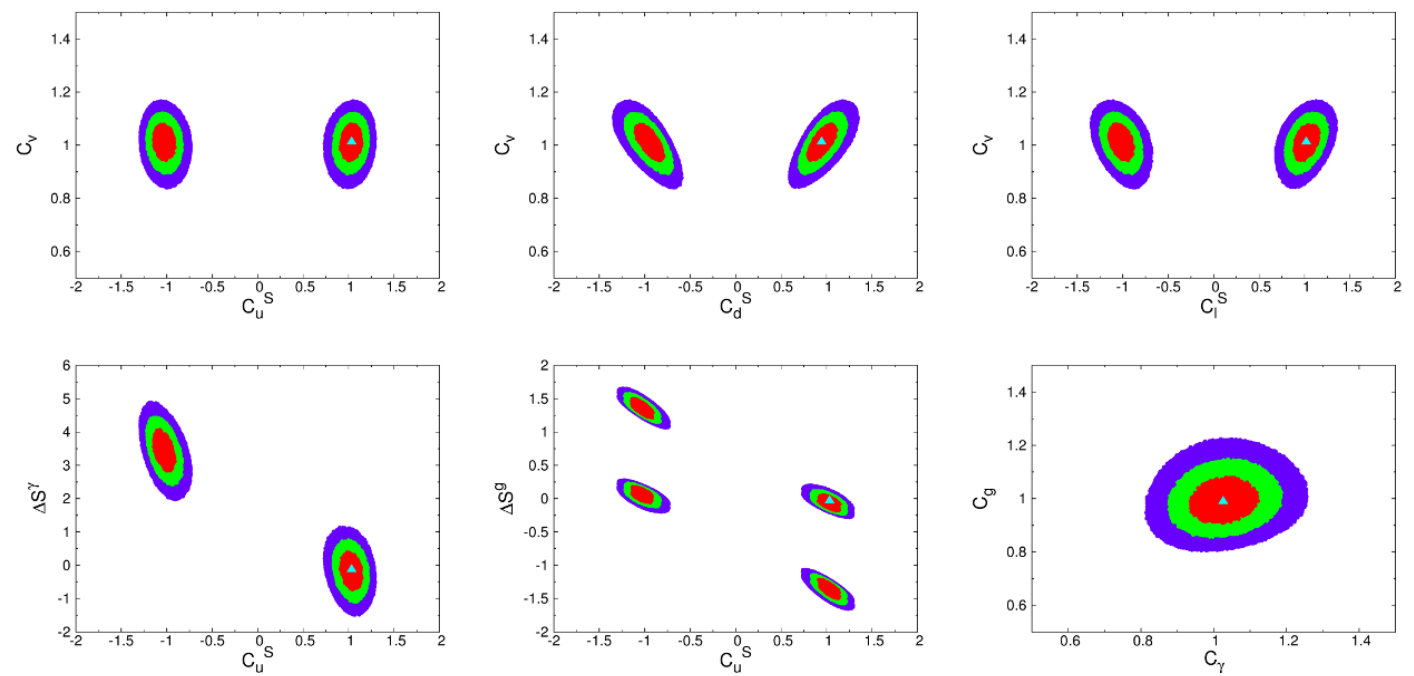

Figure 5. CPC6: the confidence-level regions of the fit by varying $\Delta S^{\gamma}, \Delta S^{g}, C_{v}, C_{u}^{S}, C_{d}^{S}$, and $C_{\ell}^{S}$. The color code is the same as figure 2 .

coupling, the vertex factor $S^{\gamma}$ only changes by $0.03 / 6.64=0.0045$, which is too small compared with experimental uncertainty. On the other hand, the vertex factor $S^{g}$ changes by $0.074 / 0.651=0.11$, which now becomes comparable to experimental uncertainty. This is the reason why the positive bottom-Yukawa is more preferred in the scenario with $\Delta S^{g}=0$. Yet, the current data precision still do not show any preference for the sign of tau-Yukawa coupling, as shown in the right panels of figure 4.

CPC6 is the most general scenario that we consider. Now confidence regions, as in the upper panels of figure 5 , show that both signs $( \pm 1)$ of top-Yukawa $C_{u}^{S}$, bottom-Yukawa $C_{d}^{S}$, and tau-Yukawa $C_{\ell}^{S}$ are equally good in describing the data, because of the compensations from $\Delta S^{g}$ and $\Delta S^{\gamma}$. For the positive sign of $C_{u}^{S}$, there are 4 possible combinations of $C_{d}^{S}$ and $C_{\ell}^{S}$ with $\Delta S^{\gamma} \sim 0,{ }^{5}$ see the lower-left panel of figure 5 . Together with the two minima at $\Delta S^{g}=-0.03(-0.10)$ and $-1.32(-1.39)$ for $C_{d}^{S} \sim 1(-1)$ as shown in lower-middle panel of figure 5, one has 8 minima. Similarly, for the negative sign of $C_{u}^{S}$, there are also 8 minima with $\Delta S^{\gamma} \sim 3.4$. In total, therefore, there are 16 degenerate minima in the CPC6 fit. In table 5, we only show the minimum at $C_{u, d, \ell}^{S} \sim 1$ and $\Delta S^{\gamma, g} \sim 0$. A substantial improvement from previous results is that the confidence-level regions shown in figure 5 are now well separated islands, while in previous results [4] those islands are "connected". For example, in the plane of $\left(C_{u}^{S}, C_{v}\right)$, the negative and positive islands of $C_{u}^{S}$ are now separated but they were connected in previous results. It means that previously $C_{u}^{S}=0$ was allowed but not in the current data.

Before moving to CPCN fits, we note that the negative top-quark Yukawa coupling is allowed only in the presence of non-zero $\Delta S^{\gamma}$ which can offset the flipped top-quark contribution to $S^{\gamma}$. The required tuning is now $\delta\left(\Delta S^{\gamma}\right) \simeq \pm 0.4$ at $1 \sigma$ level, which is about $10 \%$ of the change in $\Delta S^{\gamma}$ due to the negative top-quark Yukawa coupling. The tuning will be more and more severe as more data accumulate.

\footnotetext{
${ }^{5}$ In this work, we neglect the other possibility of $\Delta S^{\gamma} \sim 13(17)$ for positive (negative) $C_{u}^{S}$.
} 


\begin{tabular}{|c|c|c|c|c|c|c|}
\hline Cases & CPCN2 & CPCN3 & \multicolumn{4}{|c|}{ CPCN4 } \\
\hline Parameters & Vary $C_{u}^{S}, C_{v}$ & $\begin{array}{c}\text { Vary } C_{u}^{S}, C_{v} \\
\Delta S^{\gamma}\end{array}$ & \multicolumn{4}{|c|}{$\begin{array}{c}\text { Vary } C_{u}^{S}, C_{v} \\
\Delta S^{\gamma}, \Delta S^{g}\end{array}$} \\
\hline \multicolumn{7}{|c|}{ After ICHEP 2018} \\
\hline$C_{u}^{S}$ & $1.017_{-0.037}^{+0.039}$ & $1.016_{-0.038}^{+0.039}$ & $1.042_{-0.081}^{+0.077}$ & $1.042_{-0.081}^{+0.078}$ & $-1.042_{-0.078}^{+0.081}$ & $-1.042_{-0.078}^{+0.081}$ \\
\hline$C_{d}^{S}$ & 1 & 1 & 1 & 1 & 1 & 1 \\
\hline$C_{\ell}^{S}$ & 1 & 1 & 1 & 1 & 1 & 1 \\
\hline$C_{v}$ & $1.030_{-0.028}^{+0.028}$ & $1.025_{-0.035}^{+0.034}$ & $1.027_{-0.036}^{+0.034}$ & $1.027_{-0.036}^{+0.034}$ & $1.028_{-0.036}^{+0.034}$ & $1.028_{-0.036}^{+0.034}$ \\
\hline$\Delta S^{\gamma}$ & 0 & $-0.090_{-0.36}^{+0.36}$ & $-0.129_{-0.37}^{+0.37}$ & $-0.129_{-0.37}^{+0.37}$ & $3.524_{-0.42}^{+0.41}$ & $3.523_{-0.42}^{+0.41}$ \\
\hline$\Delta S^{g}$ & 0 & 0 & $-0.021_{-0.055}^{+0.057}$ & $-1.34_{-0.065}^{+0.066}$ & $0.095_{-0.057}^{+0.055}$ & $1.414_{-0.066}^{+0.066}$ \\
\hline$\Delta \Gamma_{\text {tot }}(\mathrm{MeV})$ & 0 & 0 & 0 & 0 & 0 & 0 \\
\hline$\chi^{2} / \operatorname{dof}$ & $51.16 / 62$ & $51.10 / 61$ & \multicolumn{4}{|c|}{$50.96 / 60$} \\
\hline goodness of fit & 0.835 & 0.813 & \multicolumn{4}{|c|}{0.791} \\
\hline$p$-value & 0.266 & 0.439 & \multicolumn{4}{|c|}{0.583} \\
\hline
\end{tabular}

Table 6. CPCN: the best-fitted values in various CP conserving fits and the corresponding chisquare per degree of freedom and goodness of fit. The $p$-value for each fit hypothesis against the $\mathrm{SM}$ null hypothesis is also shown. For the SM, we obtain $\chi^{2}=53.81, \chi^{2} / \operatorname{dof}=53.81 / 64$, and so the goodness of fit $=0.814$.

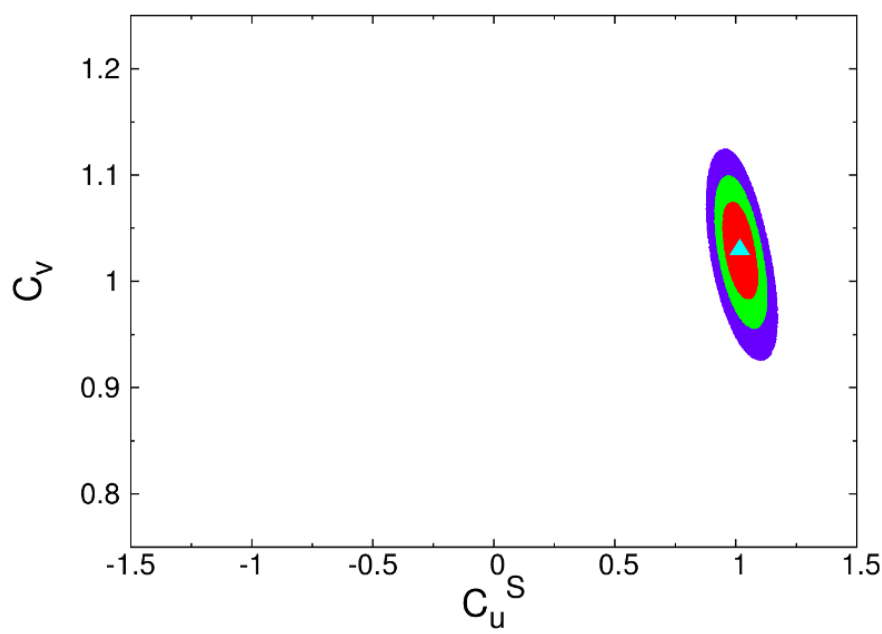

Figure 6. CPCN2: the confidence-level regions of the fit by varying $C_{u}^{S}$ and $C_{v}$. The color code is the same as in figure 2 .

\subsubsection{CPCN2 to CPCN4}

We can see in CPC2 to CPC6, the bottom-Yukawa and tau-Yukawa couplings are not very sensitive to the overall fits, though the bottom-Yukawa shows slight preference on the positive side in CPC4. Here we attempt to use the more effective parameters in the fits. The best-fits points and their $p$-values are shown in table 6 , and their corresponding figures in figure 6 to 8 .

In CPCN2, we vary only $C_{v}$ and $C_{u}^{S}$. This fit offers a slightly better $p$-value than the SM. While in CPCN3, we also vary $\Delta S^{\gamma}$ in addition to $C_{v}$ and $C_{u}^{S}$. We find that it 

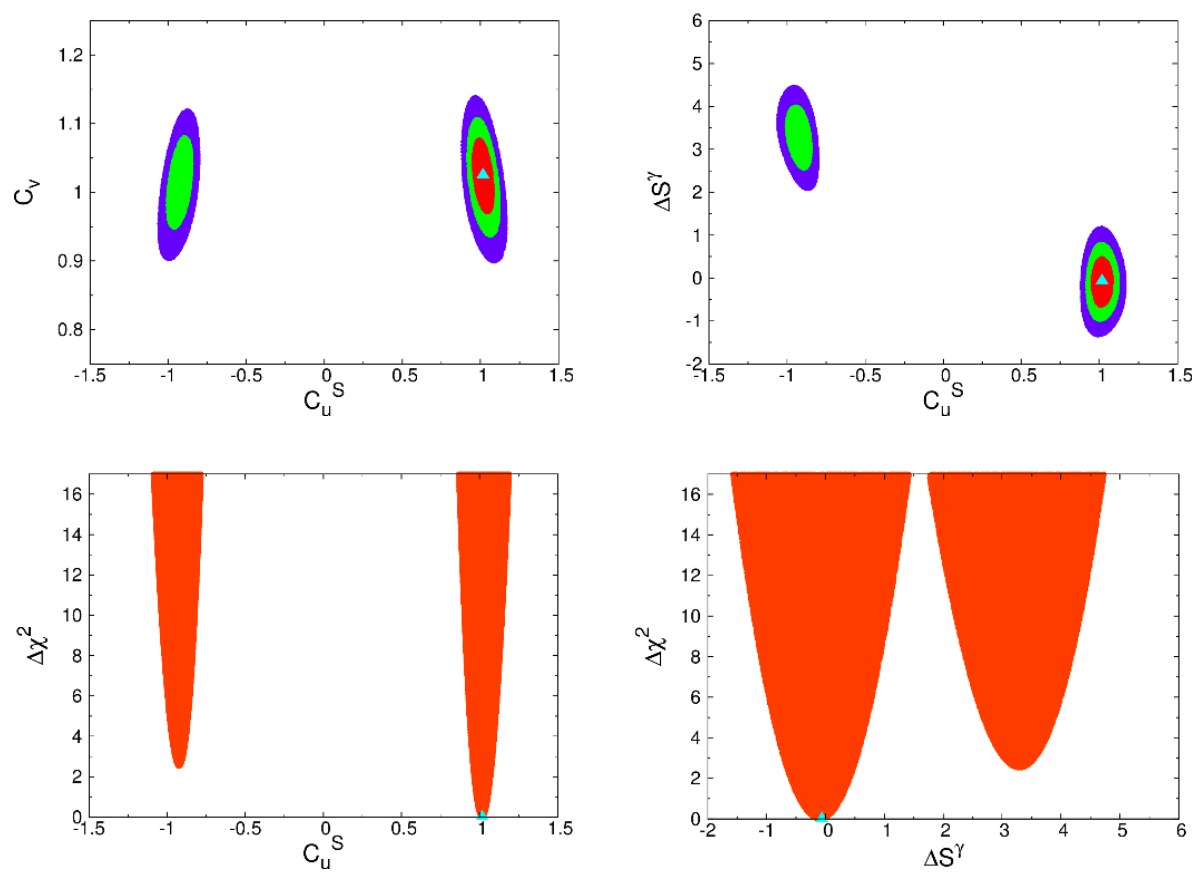

Figure 7. CPCN3: (Upper) The confidence-level regions of the fit by varying $\Delta S^{\gamma}, C_{u}^{S}$, and $C_{v}$. The color code is the same as in figure 2. (Lower) $\Delta \chi^{2}$ versus $C_{u}^{S}$ (left) and $\Delta \chi^{2}$ versus $\Delta S^{\gamma}$ (right).

has little improvement over the CPCN2 in terms of total $\chi^{2}$ but, with one less degree of freedom, the $p$-value indeed decreases. As shown in figure 7, there are two minima: the minimum near $\left(C_{u}^{S}, \Delta S^{\gamma}\right)=(1,0)$ provides a better solution by $\Delta \chi^{2} \approx 2$ than the other one near $\left(C_{u}^{S}, \Delta S^{\gamma}\right)=(-1,3.2)$. The $\Delta S^{\gamma}$ can compensate the flip in sign of $C_{u}^{S}$ in the vertex factor $S^{\gamma}$. However, when $C_{u}^{S}$ flips the sign, $\left|S^{g}\right|$ increases by more than $10 \%$ leading to a worse fit.

In CPCN4, we vary the four most efficient fitting parameters $C_{v}, C_{u}^{S}, \Delta S^{\gamma}$, and $\Delta S^{g}$. Therefore, in contrast to CPCN3, the $\Delta S^{g}$ here can compensate the sign change in $C_{u}^{S}$, such that there are four minima in this fit with the same $p$-value, as shown in table 6 and figure 8 .

\subsubsection{CPCX2 to CPCX4}

We perform some more fits, which were not considered in our previous works. The bestfit points for CPCX2 to CPCX4 are shown in table 7 and the corresponding figures in figure 9 to figure 11.

The CPCX2 fit involves $C_{v}$ and $\Delta \Gamma_{\text {tot }}$. Both parameters shift from the corresponding $\mathrm{SM}$ values in order to enhance the signal strengths. The confidence-level regions are shown in figure 9.

In addition to $C_{v}$ and $C_{u}^{S}$ (similar to CPCN2), the CPCX3 fit also varies $\Delta S^{g}$. The result is very similar to CPCN2, but $\Delta S^{g}$ has two solutions with the same $p$-values: see figure 10. 

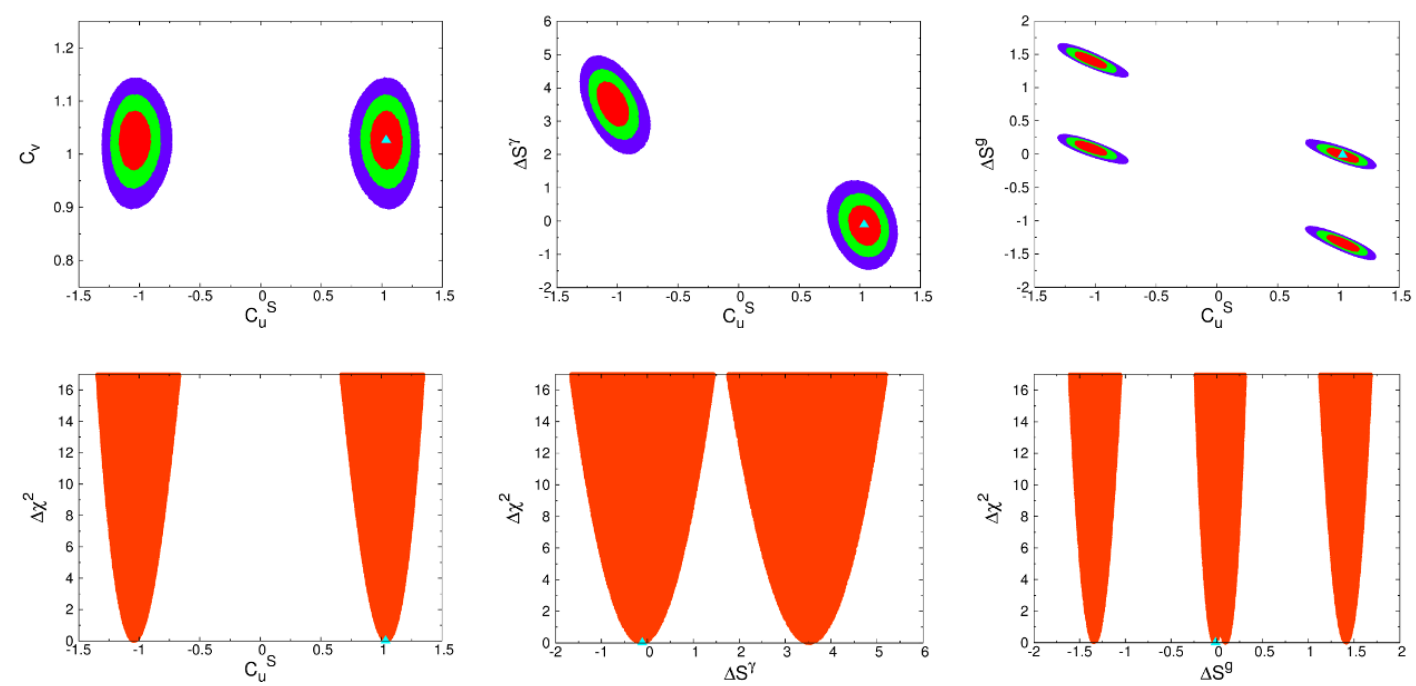

Figure 8. CPCN4: (Upper) The confidence-level regions of the fit by varying $\Delta S^{g}, \Delta S^{\gamma}, C_{u}^{S}$, and $C_{v}$. The color code is the same as in figure 2. (Lower) $\Delta \chi^{2}$ versus $C_{u}^{S}$ (left), $\Delta \chi^{2}$ versus $\Delta S^{\gamma}$ (middle), and $\Delta \chi^{2}$ versus $\Delta S^{g}$ (right).

\begin{tabular}{|c|c|c|c|c|c|c|}
\hline Cases & CPCX2 & \multicolumn{2}{|c|}{ CPCX3 } & Cases & \multicolumn{2}{|c|}{ CPCX4 } \\
\hline Parameters & Vary $C_{v}, \Delta \Gamma_{\text {tot }}$ & \multicolumn{2}{|c|}{$\begin{array}{c}\text { Vary } C_{u}^{S}, C_{v} \\
\Delta S^{g}\end{array}$} & Parameters & \multicolumn{2}{|c|}{$\begin{array}{c}\operatorname{Vary} C_{u}^{S}, C_{w} \\
C_{z}, \Delta S^{g}\end{array}$} \\
\hline \multicolumn{7}{|c|}{ After ICHEP 2018} \\
\hline$C_{u}^{S}$ & 1 & $1.04_{-0.08}^{+0.08}$ & $1.04_{-0.08}^{+0.08}$ & $C_{u}^{S}$ & $1.045_{-0.081}^{+0.078}$ & $1.045_{-0.081}^{+0.078}$ \\
\hline$C_{d}^{S}$ & 1 & 1 & 1 & $C_{d}^{S}$ & 1 & 1 \\
\hline$C_{\ell}^{S}$ & 1 & 1 & 1 & $C_{\ell}^{S}$ & 1 & 1 \\
\hline$C_{v}$ & $1.020_{-0.049}^{+0.051}$ & $1.03_{-0.03}^{+0.03}$ & $1.03_{-0.03}^{+0.03}$ & $C_{w}$ & $1.040_{-0.034}^{+0.033}$ & $1.040_{-0.034}^{+0.032}$ \\
\hline- & & & & $C_{z}$ & $1.015_{-0.049}^{+0.048}$ & $1.015_{-0.049}^{+0.048}$ \\
\hline$\Delta S^{\gamma}$ & 0 & 0 & 0 & $\Delta S^{\gamma}$ & 0 & 0 \\
\hline$\Delta S^{g}$ & 0 & $-0.02_{-0.05}^{+0.06}$ & $-1.34_{-0.06}^{+0.07}$ & $\Delta S^{g}$ & $-0.020_{-0.054}^{+0.056}$ & $-1.345_{-0.067}^{+0.067}$ \\
\hline$\Delta \Gamma_{\text {tot }}(\mathrm{MeV})$ & $-0.134_{-0.36}^{+0.43}$ & 0 & 0 & $\Delta \Gamma_{\text {tot }}(\mathrm{MeV})$ & 0 & 0 \\
\hline$\chi^{2} / d o f$ & $51.25 / 62$ & \multirow{3}{*}{\multicolumn{2}{|c|}{$\begin{array}{c}51.08 / 61 \\
0.813 \\
0.435\end{array}$}} & $\chi^{2} / d o f$ & \multirow{3}{*}{\multicolumn{2}{|c|}{$\begin{array}{c}50.84 / 60 \\
0.820 \\
0.5631\end{array}$}} \\
\hline goodness of fit & 0.833 & & & goodness of fit & & \\
\hline$p$-value & 0.278 & & & $p$-value & & \\
\hline
\end{tabular}

Table 7. CPCX: the best-fitted values in various CP conserving fits and the corresponding chisquare per degree of freedom and goodness of fit. The $p$-value for each fit hypothesis against the SM null hypothesis is also shown.

In the CPCX4 fit, we relax the requirement of $C_{w}=C_{z}$ because we can see from the $13 \mathrm{TeV}$ data that the signal strengths for $H \rightarrow W W^{*}$ are generically larger than those for $H \rightarrow Z Z^{*}$, see table 3 . The result is shown in table 7 . The best-fitted values for $C_{w}$ and $C_{z}$ are within $1 \sigma$ and $C_{w}>C_{z}$ as demanded by the data. Again there are two solutions for $\Delta S^{g}$ : see figure 11 . We note that, compared to $C_{z}$ which is only constrained by $H \rightarrow Z Z^{*}$ decay, $C_{w}$ is constrained by both $\mathrm{VBF}$ and $\mathrm{WH}$ production as well as $H \rightarrow \gamma \gamma$ 


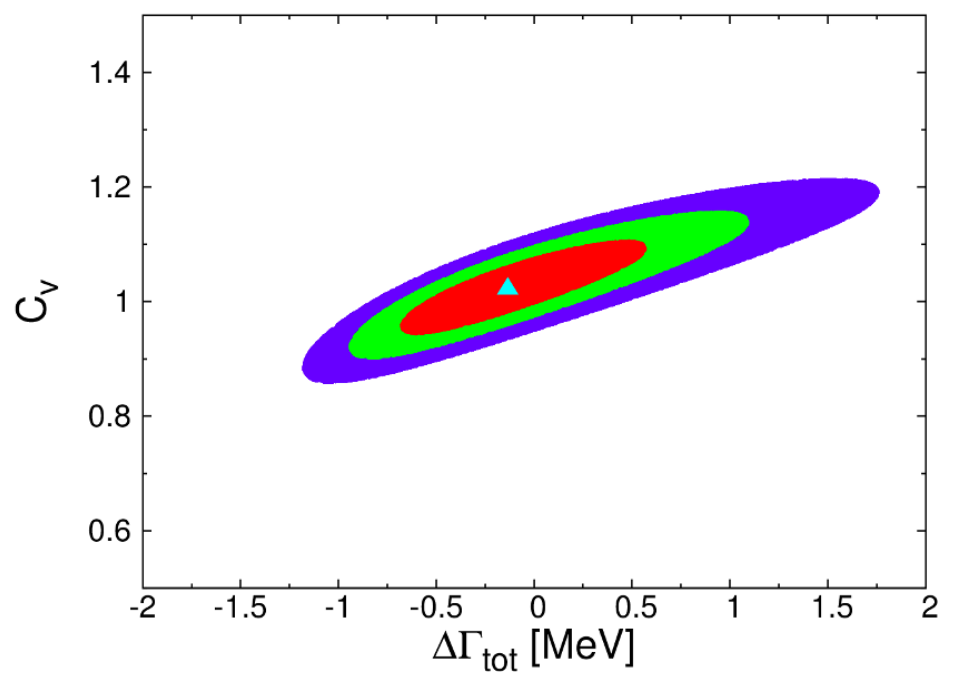

Figure 9. CPCX2: the confidence-level regions of the fit by varying $C_{v}$ and $\Delta \Gamma_{\text {tot }}$. The color code is the same as in figure 2 .
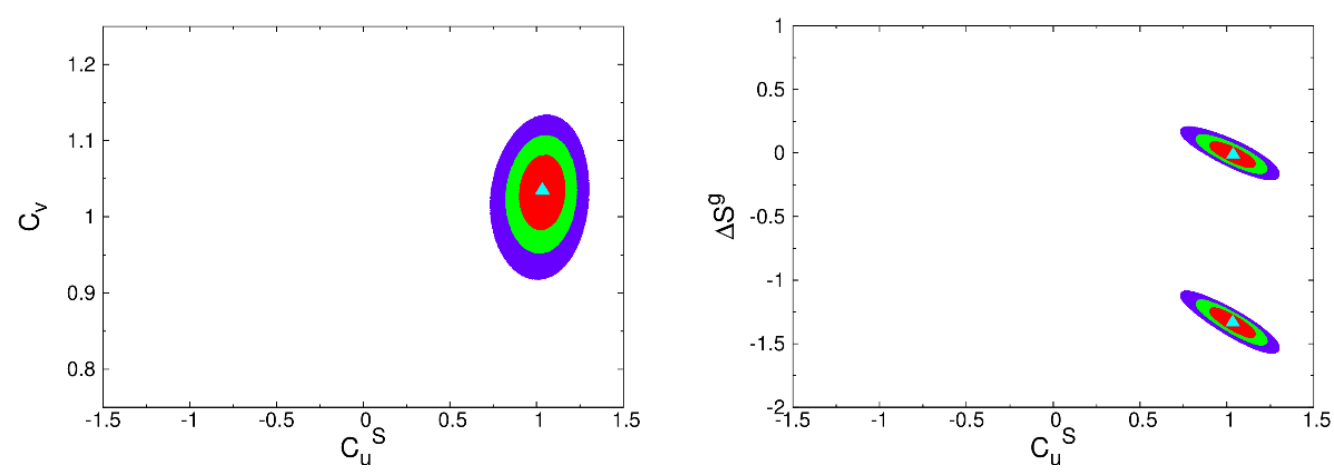

Figure 10. CPCX3: the confidence-level regions of the fit by varying $C_{v}, C_{u}^{S}$ and $\Delta S^{g}$. The color code is the same as in figure 2 .

and $H \rightarrow W W^{*}$ decays. This leads to the narrower $\Delta \chi^{2}$ curve in $C_{w}$ than in $C_{z}$, as shown in lower frames of figure 11.

\subsection{CP violating fits}

For the CP-violating fits, we consider the following 4 scenarios:

- CPV2: vary $C_{u}^{S}, C_{u}^{P}$.

- CPV3: vary $C_{u}^{S}, C_{u}^{P}, C_{v}$.

- CPV4: vary $\Delta S^{\gamma}, \Delta S^{g}, \Delta P^{\gamma}, \Delta P^{g}$.

- CPVN3: vary $C_{u}^{S}, C_{u}^{P}, \Delta \Gamma_{\text {tot }}$.

The current Higgs boson data ruled out a pure pseudoscalar [20, 21], but the data cannot rule out a mixed state [22]. Noting that the CP-odd coupling to gauge bosons only arises 

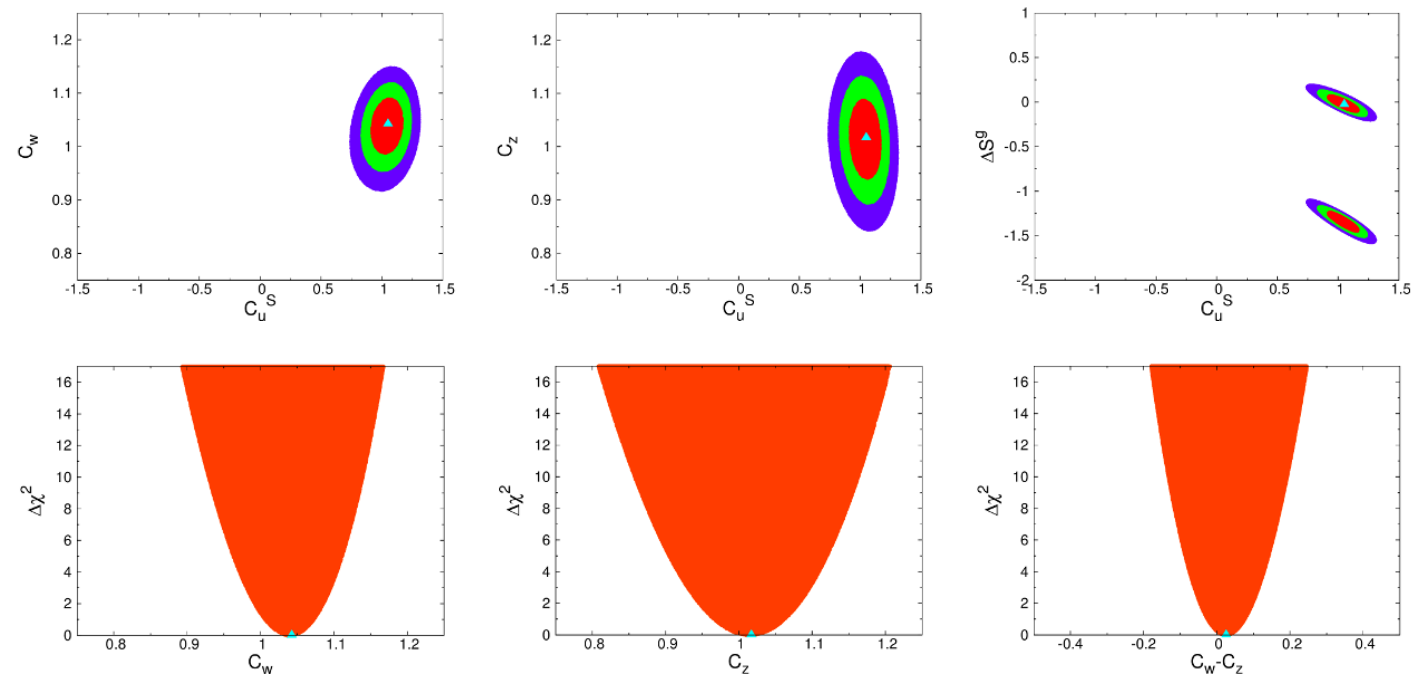

Figure 11. CPCX4: (Upper) The confidence-level regions of the fit by varying $C_{w}, C_{z}, \Delta S^{g}$, and $C_{u}^{S}$. The color code is the same as in figure 2. (Lower) $\Delta \chi^{2}$ versus $C_{w}$ (left), $\Delta \chi^{2}$ versus $C_{z}$ (middle), and $\Delta \chi^{2}$ versus $C_{w}-C_{z}$ (right).

\begin{tabular}{|c|c|c|c|c|c|c|}
\hline Cases & \multicolumn{2}{|c|}{ CPV2 } & CPV3 & CPV4 & \multicolumn{2}{|c|}{ CPVN3 } \\
\hline Parameters & \multicolumn{2}{|c|}{ Vary $C_{u}^{S}, C_{u}^{P}$} & $\begin{array}{c}\text { Vary } C_{u}^{S}, C_{u}^{P} \\
C_{v}\end{array}$ & $\begin{array}{c}\text { Vary } \Delta S^{\gamma}, \Delta S^{g} \\
\Delta P^{\gamma}, \Delta P^{g}\end{array}$ & \multicolumn{2}{|c|}{$\begin{array}{c}\text { Vary } C_{u}^{S}, C_{u}^{P} \\
\Delta \Gamma_{\text {tot }}\end{array}$} \\
\hline \multicolumn{7}{|c|}{ After ICHEP 2018} \\
\hline$C_{u}^{S}$ & $1.00_{-0.11}^{+0.07}$ & $1.00_{-0.11}^{+0.07}$ & $1.02_{-0.10}^{+0.04}$ & 1 & $0.99_{-0.10}^{+0.07}$ & $0.99_{-0.10}^{+0.07}$ \\
\hline$C_{d}^{S}$ & 1 & 1 & 1 & 1 & 1 & 1 \\
\hline$C_{\ell}^{S}$ & 1 & 1 & 1 & 1 & 1 & 1 \\
\hline$C_{v}$ & 1 & 1 & $1.03_{-0.03}^{+0.03}$ & 1 & 1 & 1 \\
\hline$\Delta S^{\gamma}$ & 0 & 0 & 0 & $0.26_{-0.81}^{+13.56}$ & 0 & 0 \\
\hline$\Delta S^{g}$ & 0 & 0 & 0 & $0.016_{-}^{+0.025}$ & 0 & 0 \\
\hline$\Delta \Gamma_{\text {tot }}(\mathrm{MeV})$ & 0 & 0 & 0 & 0 & $-0.27_{-0.28}^{+0.34}$ & $-0.27_{-0.28}^{+0.34}$ \\
\hline$C_{u}^{P}$ & $0.19_{-0.52}^{+0.14}$ & $-0.19_{-0.14}^{+0.52}$ & $0.00_{-0.28}^{+0.28}$ & 0 & $0.11_{-0.41}^{+0.19}$ & $-0.11_{-0.19}^{+0.41}$ \\
\hline$\Delta P^{\gamma}$ & 0 & 0 & 0 & $-2.54_{-4.65}^{+9.72}$ & 0 & 0 \\
\hline$\Delta P^{g}$ & 0 & 0 & 0 & $0.00_{-0.69}^{+0.69}$ & 0 & 0 \\
\hline$\chi^{2} / d o f$ & \multicolumn{2}{|c|}{$52.07 / 62$} & $51.16 / 61$ & $51.87 / 60$ & \multicolumn{2}{|c|}{$51.42 / 61$} \\
\hline goodness of fit & \multicolumn{2}{|c|}{0.812} & 0.811 & 0.763 & \multicolumn{2}{|c|}{0.804} \\
\hline$p$-value & \multicolumn{2}{|c|}{0.419} & 0.449 & 0.747 & \multicolumn{2}{|c|}{0.495} \\
\hline
\end{tabular}

Table 8. CPV: the best-fitted values in various CP violating fits and the corresponding chi-square per degree of freedom and goodness of fit. The $p$-value for each fit hypothesis against the SM null hypothesis is also shown. 


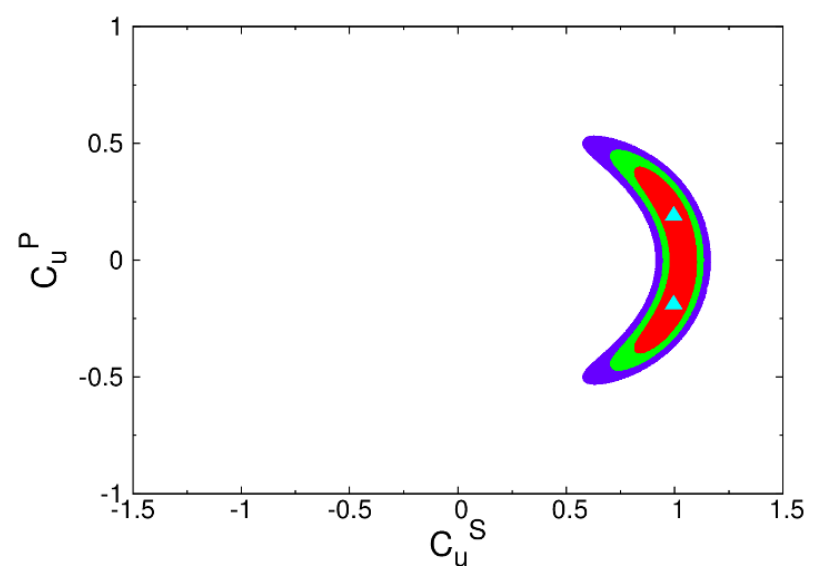

Figure 12. CPV2: the confidence-level regions of the fit by varying $C_{u}^{S}$ and $C_{u}^{P}$. The color code is the same as in figure 2 .
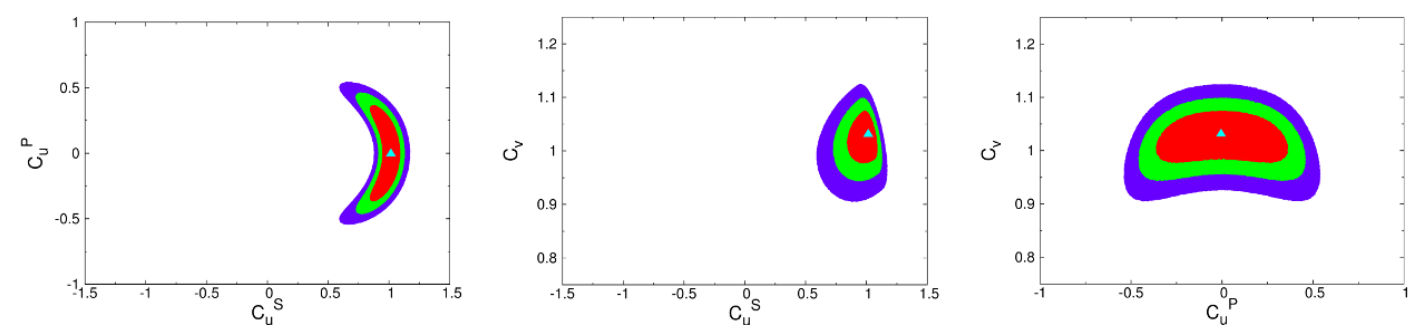

Figure 13. CPV3: the confidence-level regions of the fit by varying $C_{u}^{S}, C_{u}^{P}$, and $C_{v}$. The color code is the same as in figure 2 .
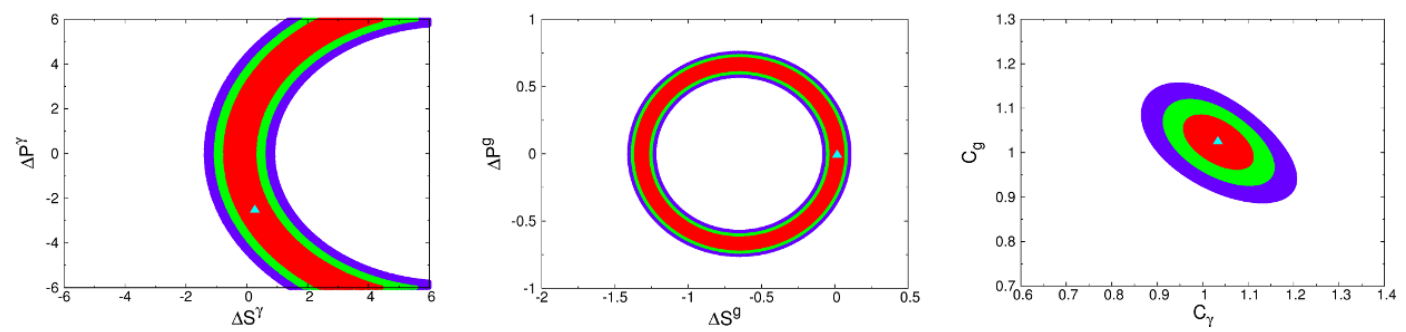

Figure 14. CPV4: the confidence-level regions of the fit by varying $\Delta S^{\gamma}, \Delta S^{g}, \Delta P^{\gamma}$, and $\Delta P^{g}$. The color code is the same as in figure 2 .

from loop corrections, we only allow the top-quark Yukawa coupling and the vertex factors for $H g g$ and $H \gamma \gamma$ to develop sizeable CP-odd couplings. Therefore, CP violation is signaled by the simultaneous existence of $C_{u}^{S}$ and $C_{u}^{P}$ as in CPV2, CPV3, and CPVN3 or of $\Delta S^{\gamma, g}$ and $\Delta P^{\gamma, g}$ in CPV4. The results for CPV2 to CPVN3 are shown in table 8 and the corresponding figures in figure 12 to figure 15.

The simplest choice CPV2 happens in the coexistence of CP-even and CP-odd topYukawa couplings: $C_{u}^{S}$ and $C_{u}^{P}$. Since the signal strengths are CP-even quantities, in general, they do not contain any CP-odd products of $C_{u}^{S} \times C_{u}^{P}$ and $S^{g, \gamma} \times P^{g, \gamma}$ even though the products are non-vanishing. This is why the confidence-level regions appear like a circle 

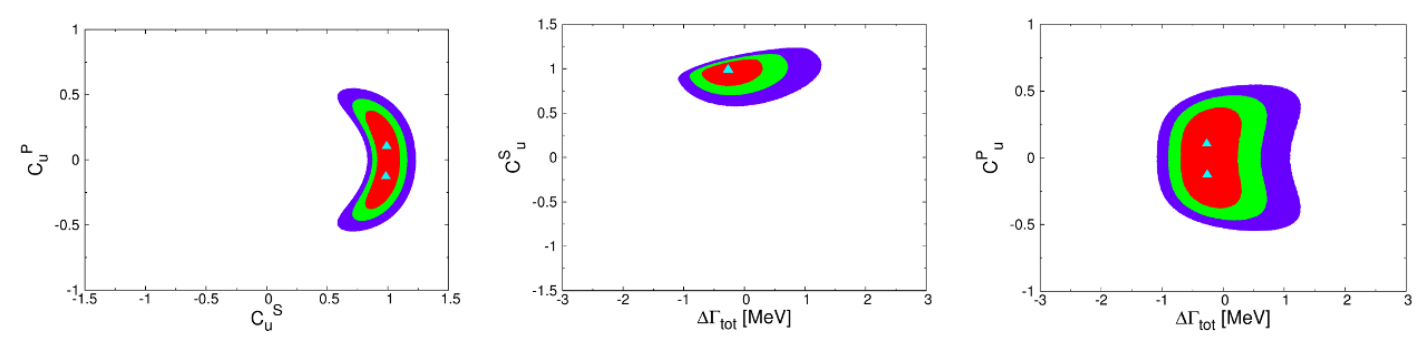

Figure 15. CPVN3: the confidence-level regions of the fit by varying $C_{u}^{S}, C_{u}^{P}$, and $\Delta \Gamma_{\text {tot }}$. The color code is the same as in figure 2 .

or an arc of a circle in the planes of $\left(C_{u}^{S}, C_{u}^{P}\right),\left(\Delta S^{\gamma}, \Delta P^{\gamma}\right)$, and $\left(\Delta S^{g}, \Delta P^{g}\right)$. In figure 12, we show two best-fit points with equal $p$-value for CPV2, indeed the arc joining these two points essentially has the same $p$-value.

We vary $C_{u}^{S}, C_{u}^{P}$, and $C_{v}$ in CPV3 fit. The confidence-level regions, shown in figure 13, shrink a lot from previous results [4]. Previously, the blue region forms a closed ellipse, but now all regions hardly form a closed ellipse, showing the data are getting much more stringent than before.

We vary $\Delta S^{g}, \Delta S^{\gamma}, \Delta P^{g}$, and $\Delta P^{\gamma}$ in CPV4. As explained in our previous work [3], the solutions to $\Delta S^{g}$ and $\Delta P^{g}$, as well as to $\Delta S^{\gamma}$ and $\Delta P^{\gamma}$ appear to be an ellipse. It is quite clear in figure 14. The best-fit points are in fact an arc inside the red region that passed through the triangle. Note that we are not considering the scenarios with too large values of $\left|\Delta S^{\gamma}\right|$ in our fits. Otherwise, the left frame of figure 14 may complete an ellipse as in the middle frame.

In the CPVN3 fit, we try a different combination of parameters: $C_{u}^{S}, C_{u}^{P}$, and $\Delta \Gamma_{\text {tot }}$. With the help of $\Delta \Gamma_{\text {tot }}$ the "banana" shaped regions originally in CPV2 now become fattened.

\subsection{Predictions for $H \rightarrow Z \gamma$}

Before we close this section, we examine how large $C_{Z \gamma}$ can be in the scenarios with $\Delta S^{\gamma}=0$, assuming the absence of additional particles running in the $H-\gamma-Z$ loop. The results are shown in figure 16 . We observe that $C_{Z \gamma}$ can be as large as 1.2 which may imply $B(H \rightarrow Z \gamma) \lesssim 1.4 B\left(H_{\mathrm{SM}} \rightarrow Z \gamma\right)$.

\section{Conclusions}

We have performed global fits to the Higgs couplings to gauge bosons and fermions, using all the data from the Tevatron, $7+8 \mathrm{TeV}$ and $13 \mathrm{TeV}$ data from ATLAS and CMS. Overall, the allowed parameter space regions shrink substantially from those in 2014. Notably, the data precision is now sensitive to the bottom-Yukawa coupling and the overall average signal strength shows a $2-\sigma$ deviation from the SM value.

Let us summarize the major findings or improvements from previous results.

1. The combined average signal strength for the Higgs boson now stands at a $2-\sigma$ deviation from the SM value, namely $\mu_{\exp }=1.10 \pm 0.05$. 

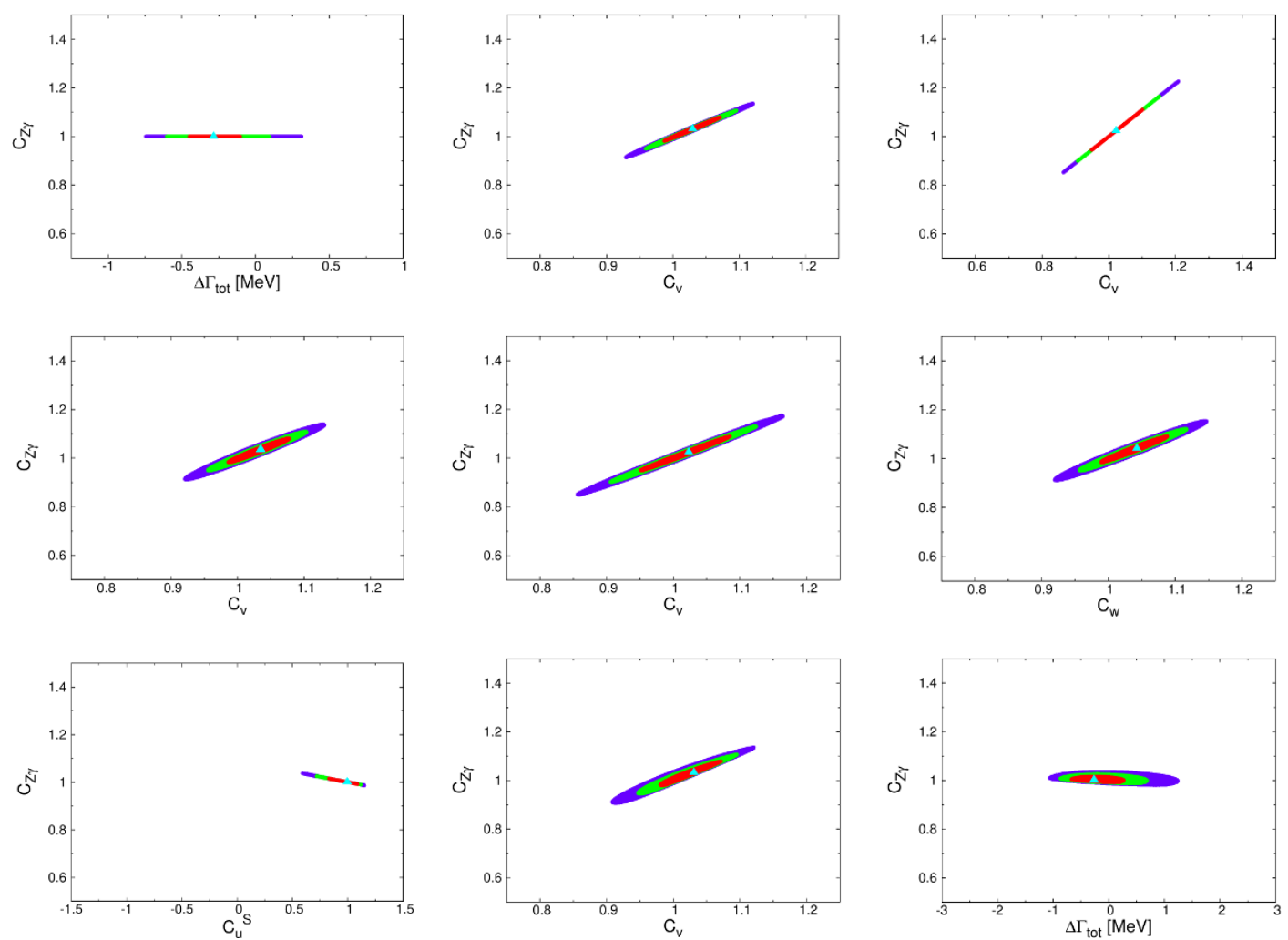

Figure 16. Predictions for $C_{Z \gamma}$ for the scenarios in which $\Delta S^{\gamma}=0$. [Upper]: CPC1 (left), CPCN2 (middle), CPCX2 (right); [Middle]: CPCX3 (left), CPC4 (middle), CPCX4 (right); [Low]: CPV2 (left), CPV3 (middle), CPVN3 (right). The color code is the same as in figure 2 except CPC1 for which $\Delta \chi^{2} \leq 1$ (red), 4 (green), and 9 (blue) above the minimum.

2. For the first time the bottom-Yukawa coupling shows statistical difference between the positive and negative signs. Thanks to the discriminating power of the Higgsgluon vertex $S^{g}$ the positive sign of the bottom-Yukawa is more preferred than the negative one.

3. Previously in 2014 the fits still allowed the negative sign of the top-Yukawa coupling at $95 \%$ CL. Now with more precisely measured signal strengths together with the establishment of the associated production with the top-quark pair, the negative island of the top-Yukawa is now entirely ruled out, except in the scenarios with nonzero $\Delta S^{\gamma}$.

4. The nonstandard (or invisible decay) branching ratio of the Higgs boson is now reduced to less than $8.4 \%$, which improves substantially from the previous value of $19 \%$. This is obtained by varying only $\Delta \Gamma_{\text {tot }}$. It would be relaxed if more parameters are allowed to vary in the fit.

5. When we relax the custodial symmetry requirement $\left(C_{w}\right.$ not necessarily equal to $C_{z}$ ), we find that the coupling $C_{w}$ is larger than $C_{z}$ though still within $1 \sigma$, and more constrained than $C_{z}$. 
6. We have also made the predictions for $H \rightarrow Z \gamma$ by showing the effective coupling $C_{Z \gamma}$. In most scenarios, it is predicted to be SM-like. The most extreme allowed value would $C_{Z \gamma} \simeq 1.2$, which gives a branching ratio $40 \%$ larger than the $\mathrm{SM}$ value.

\section{Acknowledgments}

The work of K.C. was supported by the National Science Council of Taiwan under Grants Nos. MOST-105-2112-M-007-028-MY3 and MOST-107-2112-M-007-029-MY3. The work of J.S.L. was supported by the National Research Foundation of Korea (NRF) grant No. NRF-2016R1E1A1A01943297. The work of P.-Y.T. was supported by World Premier International Research Center Initiative (WPI), MEXT, Japan. We thank Jubin Park for useful discussions.

\section{A Relation between our formalism and the kappa formalism}

Here in this appendix we compare the definitions of coupling modifiers taken in our formalism to those defined by LHCHXSWG, and make the correspondence taking the specific examples of the loop-induced $H g g$ and $H \gamma \gamma$ couplings.

In the LHCHXSWG YR3 [14] and YR4 [15], as well as in a very recent paper by ATLAS [16], the definition of $\kappa_{g}$ is given by

$$
\kappa_{g}^{2}\left(\kappa_{b}, \kappa_{t}, M_{H}\right) \equiv \frac{\kappa_{t}^{2} \cdot \sigma_{g g H}^{t t}\left(M_{H}\right)+\kappa_{b}^{2} \cdot \sigma_{g g H}^{b b}\left(M_{H}\right)+\kappa_{t} \kappa_{b} \cdot \sigma_{g g H}^{t b}\left(M_{H}\right)}{\sigma_{g g H}^{t t}\left(M_{H}\right)+\sigma_{g g H}^{b b}\left(M_{H}\right)+\sigma_{g g H}^{t b}\left(M_{H}\right)}
$$

where $\sigma_{g g H}^{t t}\left(M_{H}\right)$ and $\sigma_{g g H}^{b b}\left(M_{H}\right)$ denote the squares of the top and bottom contributions to the $g g \rightarrow H$ production, respectively, and $\sigma_{g g H}^{t b}\left(M_{H}\right)$ the top-bottom interference. On the other hand, $\kappa_{g}$ can be also defined through the $H \rightarrow g g$ decay process:

$$
\kappa_{g}^{2} \equiv \frac{\Gamma(H \rightarrow g g)}{\Gamma^{\mathrm{SM}}(H \rightarrow g g)} .
$$

The LHCHXSWG is performing analyses through $g g \rightarrow H$ production at $8 \mathrm{TeV}$ beyond the leading order and, taking $M_{H}=125 \mathrm{GeV}$, they find $[14,15]$

$$
\begin{aligned}
& \kappa_{g, \mathrm{YR} 3}^{2}=1.058 \kappa_{t}^{2}+0.007 \kappa_{b}^{2}-0.065 \kappa_{t} \kappa_{b} \\
& \kappa_{g, \mathrm{YR} 4}^{2}=1.042 \kappa_{t}^{2}+0.002 \kappa_{b}^{2}-0.040 \kappa_{t} \kappa_{b}-0.005 \kappa_{t} \kappa_{c}+0.0005 \kappa_{b} \kappa_{c}+0.00002 \kappa_{c}^{2} .
\end{aligned}
$$

The difference between $\kappa_{g \text {,YR3 }}^{2}$ and $\kappa_{g \text {,YR }}^{2}$ can be attributed to the choices of the QCD and factorization scales and the PDF set, the different remormalization scheme for the masses of the fermions entering into the loops, etc. On the other hand, in the recent paper by ATLAS, the simpler $\kappa_{g}^{2}$ based on the Higgs decay $H \rightarrow g g$ is taken and they find [16]:

$$
\kappa_{g, \mathrm{ATLAS}}^{2}=1.11 \kappa_{t}^{2}+0.01 \kappa_{b}^{2}-0.12 \kappa_{t} \kappa_{b}
$$

which is closer to $\kappa_{g, \mathrm{YR} 3}^{2}$. 
In our work, we only perform LO analysis and $\kappa_{g}^{2}$ is given by

$$
\kappa_{g}^{2}=C_{g}^{2}=\frac{\left|S^{g}\left(M_{H}\right)\right|^{2}+\left|P^{g}\left(M_{H}\right)\right|^{2}}{\left|S_{\mathrm{SM}}^{g}\left(M_{H}\right)\right|^{2}}
$$

independently of whether one considers $g g \rightarrow H$ production or $H \rightarrow g g$ decay. Using the numerical expression eq. (2.8) which is obtained by taking $M_{H}=125.09 \mathrm{GeV}$ and $\Delta S^{g}=P^{g}=0$, we have

$$
\kappa_{g, \text { OURS }}^{2} \simeq 1.11 C_{t}^{2}+0.01 C_{b}^{2}-0.12 C_{t} C_{b},
$$

which is very consistent with $\kappa_{g, \text { ATLAS }}^{2}$ based on $H \rightarrow g g$ decay with the identification of $C_{f}=\kappa_{f}$.

One of the important findings of our work is the preferred sign of the bottom-Yukawa coupling $C_{b}$. The observable effect on signal strength due to flipping of the sign of $C_{b}$ comes from the interference term $\left(\propto C_{t} C_{b}\right.$ or $\left.\kappa_{t} \kappa_{b}\right)$, but not the square of the bottom-Yukawa term. Thus, all three expressions of $\kappa_{g, \mathrm{YR} 3}^{2}, \kappa_{g, \mathrm{YR} 4}^{2}$, and $\kappa_{g, \mathrm{ATLAS}}^{2}$ and our expression of $\kappa_{g, \text { OURS }}^{2}$ yield a similar change of order $O(10) \%$ due to the flipping of the sign of the bottom-Yukawa coupling.

Similarly, LHCHXSWG gives the definition for $\kappa_{\gamma}$ :

$$
\kappa_{\gamma}^{2} \equiv \frac{\sum_{i, j} \kappa_{i} \kappa_{j} \Gamma_{\gamma \gamma}^{i j}\left(M_{H}\right)}{\sum_{i, j} \Gamma_{\gamma \gamma}^{i j}\left(M_{H}\right)} \simeq 1.59 \kappa_{W}^{2}+0.07 \kappa_{t}^{2}-0.67 \kappa_{W} \kappa_{t}
$$

where the pairs $(i, j)$ are $b b, t t, \tau \tau, W W, b t, b \tau, b W, t \tau, t W$, and $\tau W$. The above numerical expression is compared with that obtained by the use of eq. (2.5) after taking $M_{H}=125.09 \mathrm{GeV}$ and $\Delta S^{\gamma}=P^{\gamma}=0$ :

$$
\begin{aligned}
\kappa_{\gamma}^{2} \simeq & 1.583 C_{W}^{2}+0.070 C_{t}^{2}-0.667 C_{W} C_{t}+0.006 C_{W} C_{b} \\
& +0.009 C_{W} C_{\tau}+0.003 C_{W} C_{c}-0.001 C_{t} C_{b}-0.002 C_{t} C_{\tau},
\end{aligned}
$$

and we find excellent consistency. We note that our formalism takes on the advantage that it can admit pseudoscalar form factor $P^{g}$ and $P^{\gamma}$ into the effective $H g g$ and $H \gamma \gamma$ vertices. 


\section{B $\quad 13$ TeV data: tables $9,10,11,12,13$, and 14}

In this appendix, we list all the details of $13 \mathrm{TeV}$ Higgs signal strengths used in our global fitting.

\begin{tabular}{|c|c|c|c|}
\hline Channel & $\begin{array}{c}\text { Signal strength } \mu \\
\text { c.v } \pm \text { error }\end{array}$ & $M_{H}(\mathrm{GeV})$ & $\chi_{\mathrm{SM}}^{2}($ each $)$ \\
\hline \multicolumn{4}{|c|}{ ATLAS $\left(79.8 \mathrm{fb}^{-1}(13 \mathrm{TeV})\right)$ : figure 8 of [23] (Jul. 2018) } \\
\hline $\operatorname{ggF}$ & $0.97_{-0.14}^{+0.15}[23]$ & 125.09 & 0.04 \\
\hline $\mathrm{VBF}$ & $1.40_{-0.37}^{+0.43}[23]$ & 125.09 & 1.17 \\
\hline $\mathrm{VH}$ & $1.08_{-0.54}^{+0.59}[23]$ & 125.09 & 0.02 \\
\hline $\mathrm{ttH}$ & $1.12_{-0.37}^{+0.43}[23]$ & 125.09 & 0.11 \\
\hline \multicolumn{4}{|c|}{ CMS $\left(35.9 \mathrm{fb}^{-1}(13 \mathrm{TeV})\right)$ : figure 17 of [24] (Apr. 2018) } \\
\hline $\operatorname{ggF}$ & $1.10_{-0.18}^{+0.20}[24]$ & 125.4 & 0.31 \\
\hline VBF & $0.8_{-0.5}^{+0.6}[24]$ & 125.4 & 0.11 \\
\hline $\mathrm{VH}$ & $2.4_{-1.0}^{+1.1}[24]$ & 125.4 & 1.96 \\
\hline \multirow[t]{2}{*}{$\mathrm{ttH}$} & $2.2_{-0.8}^{+0.9}[24]$ & 125.4 & 2.25 \\
\hline & & & subtot: 5.97 \\
\hline
\end{tabular}

Table 9. ( $L H C: 13 \mathrm{TeV}$ ) Data on signal strengths of $H \rightarrow \gamma \gamma$ by the ATLAS and CMS after ICHEP 2018. The $\chi^{2}$ of each data with respect to the SM is shown in the last column. The sub-total $\chi^{2}$ of this decay mode is shown at the end.

\begin{tabular}{|c|c|c|c|}
\hline Channel & $\begin{array}{c}\text { Signal strength } \mu \\
\text { c.v } \pm \text { error }\end{array}$ & $M_{H}(\mathrm{GeV})$ & $\chi_{\mathrm{SM}}^{2}($ each $)$ \\
\hline \multicolumn{4}{|c|}{$\operatorname{ATLAS}\left(79.8 \mathrm{fb}^{-1}\right.$ at $\left.13 \mathrm{TeV}\right)$ : table 9 of [25] (Jun. 2018) } \\
\hline $\operatorname{ggF}$ & $1.04_{-0.16}^{+0.16}[25]$ & 125 & 0.06 \\
\hline VBF & $2.8_{-0.94}^{+0.94}[25]$ & 125 & 3.67 \\
\hline VH & $0.9_{-1.01}^{+1.01}[25]$ & 125 & 0.01 \\
\hline $\mathrm{ttH}^{\dagger}$ & $<4.04(95 \%)[25]$ & 125 & - \\
\hline \multicolumn{4}{|c|}{ CMS (77.4 $\mathrm{fb}^{-1}$ at $\left.13 \mathrm{TeV}\right)$ : figure 10 of [26] (Jul. 2018) } \\
\hline $\operatorname{ggF}$ & $1.15_{-0.16}^{+0.18}[26]$ & 125.09 & 0.88 \\
\hline VBF & $0.69_{-0.57}^{+0.75}[26]$ & 125.09 & 0.17 \\
\hline $\mathrm{VH}_{\text {had }}$ & $0.00_{-0.00}^{+1.16}[26]$ & 125.09 & 0.74 \\
\hline $\mathrm{VH}_{\text {lep }}$ & $1.25_{-1.25}^{+2.46}[26]$ & 125.09 & 0.04 \\
\hline \multirow[t]{2}{*}{$\mathrm{ttH}$} & $0.00_{-0.00}^{+0.53}[26]$ & 125.09 & 3.56 \\
\hline & & & subtot: 9.13 \\
\hline
\end{tabular}

Table 10. (LHC: $13 \mathrm{TeV}$ ) The same as table 9 but for $H \rightarrow Z Z^{(*)}$. 


\begin{tabular}{|cccr|}
\hline Channel & $\begin{array}{c}\text { Signal strength } \mu \\
\text { c.v } \pm \text { error }\end{array}$ & $M_{H}(\mathrm{GeV})$ & $\chi_{\text {SM }}^{2}$ (each) \\
\hline ATLAS $\left(36.1 \mathrm{fb}^{-1}(13 \mathrm{TeV})\right)$ : page 8 of $[27]$ & (Mar. 2018$)$ \\
\hline ggF & $1.21_{-0.21}^{+0.22}[27]$ & 125 & 1.00 \\
VBF & $0.62_{-0.36}^{+0.37}[27]$ & 125 & 1.05 \\
\hline CMS $\left(35.9 \mathrm{fb}^{-1}\right.$ at $\left.13 \mathrm{TeV}\right)$ : figure 9 of $[28]$ & (Mar. 2018$)$ \\
\hline ggF & $1.38_{-0.24}^{+0.21}[28]$ & 125.09 & 2.51 \\
VBF & $0.29_{-0.29}^{+0.66}[28]$ & 125.09 & 1.16 \\
WH & $3.27_{-1.70}^{+1.88}[28]$ & 125.09 & 1.78 \\
ZH & $1.00_{-1.00}^{+1.57}[28]$ & 125.09 & 0.00 \\
\hline \multicolumn{2}{c}{} & & subtot: 7.50 \\
\hline
\end{tabular}

Table 11. (LHC: $13 \mathrm{TeV}$ ) The same as table 9 but for $H \rightarrow W^{+} W^{-}$.

\begin{tabular}{|c|c|c|c|}
\hline Channel & $\begin{array}{c}\text { Signal strength } \mu \\
\text { c.v } \pm \text { error }\end{array}$ & $M_{H}(\mathrm{GeV})$ & $\chi_{\mathrm{SM}}^{2}($ each $)$ \\
\hline \multicolumn{4}{|c|}{ ATLAS $\left(79.8 \mathrm{fb}^{-1}(13 \mathrm{TeV})\right)$ figure 3 of [29] (Aug. 2018) } \\
\hline WH & $1.08_{-0.43}^{+0.47}[29]$ & 125.0 & 0.03 \\
\hline $\mathrm{ZH}$ & $1.20_{-0.31}^{+0.33}[29]$ & 125.0 & 0.42 \\
\hline \multicolumn{4}{|c|}{ CMS $\left(77.2 \mathrm{fb}^{-1}(13 \mathrm{TeV})\right)[7,30]$ (Aug. 2018) } \\
\hline $\operatorname{ggH}$ & $2.51_{-2.01}^{+2.43}[30]$ & 125.09 & 0.56 \\
\hline $\mathrm{VH}$ & $1.06_{-0.26}^{+0.26}[7]$ & 125.09 & 0.05 \\
\hline $\mathrm{ttH}$ & $0.91_{-0.43}^{+0.45}[30]$ & 125.09 & 0.04 \\
\hline
\end{tabular}

Table 12. (LHC: $13 \mathrm{TeV}$ ) The same as table 9 but for $H \rightarrow b \bar{b}$.

\begin{tabular}{|cccc|}
\hline Channel & $\begin{array}{c}\text { Signal strength } \mu \\
\text { c.v } \pm \text { error }\end{array}$ & $M_{H}(\mathrm{GeV})$ & $\chi_{\mathrm{SM}}^{2}$ (each) \\
\hline \multicolumn{4}{|c|}{ ATLAS $\left(36.1 \mathrm{fb}^{-1}\right.$ at $\left.13 \mathrm{TeV}\right)[10]$ (Jun. 2018) } \\
\hline ggH & $0.98_{-0.51}^{+0.62}[10]$ & 125.09 & 0.00 \\
$\mathrm{VBF}$ & $1.18_{-0.55}^{+0.59}[10]$ & 125.09 & 0.11 \\
\hline $\mathrm{CMS}\left(35.9 \mathrm{fb}^{-1}\right.$ at $\left.13 \mathrm{TeV}\right)$ & figure 6 of [31] & (Jun. 2018$)$ \\
\hline ggH & $1.12_{-0.50}^{+0.53}[31]$ & 125.09 & 0.06 \\
$\mathrm{VBF}$ & $1.13_{-0.42}^{+0.45}[31]$ & 125.09 & 0.10 \\
$\mathrm{WH}$ & $3.39_{-1.54}^{+1.68}[31]$ & 125.09 & 2.41 \\
$\mathrm{ZH}$ & $1.23_{-1.35}^{+1.62}[31]$ & 125.09 & 0.03 \\
\hline \multicolumn{4}{r}{} \\
\end{tabular}

Table 13. (LHC: $13 \mathrm{TeV}$ ) The same as table 9 but for $H \rightarrow \tau^{+} \tau^{-}$. 


\begin{tabular}{|cccc|}
\hline Channel & $\begin{array}{c}\text { Signal strength } \mu \\
\text { c.v } \pm \text { error }\end{array}$ & $M_{H}(\mathrm{GeV})$ & $\chi_{\mathrm{SM}}^{2}($ each $)$ \\
\hline \multicolumn{4}{|c|}{ ATLAS $\left(79.8 \mathrm{fb}^{-1}(13 \mathrm{TeV})\right)$ : figure 5 of [6] (Jun. 2018) } \\
$\left(36.1 \mathrm{fb}^{-1}(13 \mathrm{TeV})\right)$ : figure 16 of [32] (Apr. 2018)
\end{tabular}

${ }^{\dagger}$ : this data point is not included in our $\chi^{2}$ analysis.

Table 14. (LHC: $13 \mathrm{TeV}$ ) The same as table 9 but for exclusive $t t H$ production mode.

Open Access. This article is distributed under the terms of the Creative Commons Attribution License (CC-BY 4.0), which permits any use, distribution and reproduction in any medium, provided the original author(s) and source are credited.

\section{References}

[1] ATLAS collaboration, Observation of a new particle in the search for the standard model Higgs boson with the ATLAS detector at the LHC, Phys. Lett. B 716 (2012) 1 [arXiv: 1207.7214] [INSPIRE].

[2] CMS collaboration, Observation of a new boson at a mass of $125 \mathrm{GeV}$ with the CMS experiment at the LHC, Phys. Lett. B 716 (2012) 30 [arXiv:1207.7235] [INSPIRE].

[3] K. Cheung, J.S. Lee and P.-Y. Tseng, Higgs precision (Higgcision) era begins, JHEP 05 (2013) 134 [arXiv:1302.3794] [INSPIRE].

[4] K. Cheung, J.S. Lee and P.Y. Tseng, Higgs precision analysis updates 2014, Phys. Rev. D 90 (2014) 095009 [arXiv:1407.8236].

[5] CMS collaboration, Evidence for associated production of a Higgs boson with a top quark pair in final states with electrons, muons and hadronically decaying $\tau$ leptons at $\sqrt{s}=13$ TeV, JHEP 08 (2018) 066 [arXiv: 1803.05485] [INSPIRE].

[6] ATLAS collaboration, Observation of Higgs boson production in association with a top quark pair at the LHC with the ATLAS detector, Phys. Lett. B 784 (2018) 173 [arXiv: 1806.00425] [INSPIRE].

[7] CMS collaboration, Observation of Higgs boson decay to bottom quarks, Phys. Rev. Lett. 121 (2018) 121801 [arXiv:1808.08242] [INSPIRE]. 
[8] ATLAS collaboration, Evidence for the $H \rightarrow b \bar{b}$ decay with the ATLAS detector, JHEP 12 (2017) 024 [arXiv : 1708.03299] [INSPIRE].

[9] CMS collaboration, Observation of the Higgs boson decay to a pair of $\tau$ leptons with the CMS detector, Phys. Lett. B 779 (2018) 283 [arXiv:1708.00373] [INSPIRE].

[10] ATLAS collaboration, Cross-section measurements of the Higgs boson decaying to a pair of tau leptons in proton-proton collisions at $\sqrt{s}=13 \mathrm{TeV}$ with the ATLAS detector, ATLAS-CONF-2018-021 (2018).

[11] ATLAS, CMS collaboration, Measurements of the Higgs boson production and decay rates and constraints on its couplings from a combined ATLAS and CMS analysis of the LHC pp collision data at $\sqrt{s}=7$ and $8 \mathrm{TeV}$, JHEP 08 (2016) 045 [arXiv:1606.02266] [INSPIRE].

[12] A. Juste, Standard Model Higgs boson searches at the Tevatron, in the proceedings of the Hadron Collider Physics Symposium 2012 (HCP2012), November 12-16, 2012, Kyoto, Japan (2012).

[13] K. Herner, Studies of the Higgs boson properties at DØ, in the proceedings of the $37^{\text {th }}$ International Conference on High Energy Physics (ICHEP 2014), July 2-9, Valencia, Spain (2014).

[14] S. Heinemeyer et al., Handbook of LHC Higgs cross sections: 3. Higgs properties, arXiv:1307.1347 [CERN-2013-004].

[15] LHC Higgs Cross Section Working Group collaboration, Handbook of LHC Higgs cross sections: 4. Deciphering the nature of the Higgs sector, arXiv:1610.07922 [INSPIRE].

[16] ATLAS collaboration, Combined measurements of Higgs boson production and decay using up to $80 \mathrm{fb}^{-1}$ of proton-proton collision data at $\sqrt{s}=13 \mathrm{TeV}$ collected with the ATLAS experiment, ATLAS-CONF-2019-005 (2019).

[17] J.S. Lee et al., CPsuperH: a computational tool for Higgs phenomenology in the minimal supersymmetric standard model with explicit CP-violation, Comput. Phys. Commun. 156 (2004) 283 [hep-ph/0307377] [INSPIRE].

[18] J.S. Lee et al., CPsuperH2.0: an improved computational tool for Higgs phenomenology in the MSSM with explicit CP-violation, Comput. Phys. Commun. 180 (2009) 312 [arXiv:0712.2360] [INSPIRE].

[19] J.S. Lee et al., CPsuperH2.3: an updated tool for phenomenology in the MSSM with explicit CP-violation, Comput. Phys. Commun. 184 (2013) 1220 [arXiv:1208.2212] [InSPIRE].

[20] ATLAS collaboration, Evidence for the spin-0 nature of the Higgs boson using ATLAS data, Phys. Lett. B 726 (2013) 120 [arXiv:1307.1432] [INSPIRE].

[21] CMS collaboration, Constraints on the spin-parity and anomalous HVV couplings of the Higgs boson in proton collisions at 7 and 8 TeV, Phys. Rev. D 92 (2015) 012004 [arXiv: 1411.3441] [INSPIRE].

[22] ATLAS collaboration, Test of CP invariance in vector-boson fusion production of the Higgs boson using the Optimal Observable method in the ditau decay channel with the ATLAS detector, Eur. Phys. J. C 76 (2016) 658 [arXiv:1602.04516] [INSPIRE].

[23] ATLAS collaboration, Measurements of Higgs boson properties in the diphoton decay channel using $80 \mathrm{fb}^{-1}$ of pp collision data at $\sqrt{\mathrm{s}}=13 \mathrm{TeV}$ with the ATLAS detector,

ATLAS-CONF-2018-028 (2018). 
[24] CMS collaboration, Measurements of Higgs boson properties in the diphoton decay channel in proton-proton collisions at $\sqrt{s}=13 \mathrm{TeV}$, JHEP 11 (2018) 185 [arXiv:1804.02716] [INSPIRE].

[25] ATLAS collaboration, Measurements of the Higgs boson production, fiducial and differential cross sections in the $4 \ell$ decay channel at $\sqrt{s}=13$ TeV with the ATLAS detector, ATLAS-CONF-2018-018 (2018).

[26] CMS collaboration, Measurements of properties of the Higgs boson in the four-lepton final state at $\sqrt{s}=13$ TeV, CMS-PAS-HIG-18-001 (2018).

[27] ATLAS collaboration, Measurement of gluon fusion and vector boson fusion Higgs boson production cross-sections in the $H \rightarrow W W^{*} \rightarrow e \nu \mu \nu$ decay channel in pp collisions at $\sqrt{s}=13$ TeV with the ATLAS detector, ATLAS-CONF-2018-004 (2018).

[28] CMS collaboration, Measurements of properties of the Higgs boson decaying to a $W$ boson pair in pp collisions at $\sqrt{s}=13$ TeV, CMS-PAS-HIG-16-042 (2016).

[29] ATLAS collaboration, Observation of $H \rightarrow b \bar{b}$ decays and $V H$ production with the ATLAS detector, Phys. Lett. B 786 (2018) 59 [arXiv: 1808.08238] [INSPIRE].

[30] CMS Collaboration, Combined measurements of the Higgs boson's couplings at $\sqrt{s}=13 \mathrm{TeV}$, CMS-PAS-HIG-17-031 (Combined measurements of the Higgs boson's couplings at $\sqrt{s}=13$ $\mathrm{TeV})$.

[31] CMS collaboration, Search for the standard model Higgs boson decaying to a pair of $\tau$ leptons and produced in association with a $W$ or a $Z$ boson in proton-proton collisions at $\sqrt{s}=13$ TeV, CMS-PAS-HIG-18-007 (2018).

[32] ATLAS collaboration, Evidence for the associated production of the Higgs boson and a top quark pair with the ATLAS detector, Phys. Rev. D 97 (2018) 072003 [arXiv:1712.08891] [INSPIRE].

[33] ATLAS collaboration, Search for the standard model Higgs boson produced in association

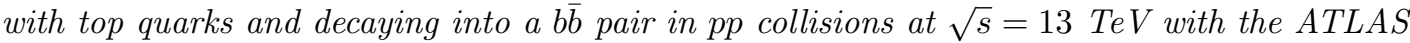
detector, Phys. Rev. D 97 (2018) 072016 [arXiv: 1712.08895] [INSPIRE].

[34] CMS collaboration, Search for $t \bar{t} H$ production in the all-jet final state in proton-proton collisions at $\sqrt{s}=13 \mathrm{TeV}$, JHEP 06 (2018) 101 [arXiv: 1803.06986] [INSPIRE].

[35] CMS collaboration, Search for $t \bar{t} H$ production in the $H \rightarrow b \bar{b}$ decay channel with leptonic $t \bar{t}$ decays in proton-proton collisions at $\sqrt{s}=13$ TeV, JHEP 03 (2019) 026 [arXiv:1804.03682] [INSPIRE]. 